Article

\title{
A Comparison of Techniques for Reducing Unicast Traffic in HSR Networks
}

\author{
Nguyen Xuan Tien, Saad Allawi Nsaif and Jong Myung Rhee * \\ Department of Information and Communications Engineering, Myongji University, Yongin, \\ Gyeonggi 449-728, Korea; E-Mails: nxtien@gmail.com (N.X.T.); saad.allawi1@gmail.com (S.A.N.) \\ * Author to whom correspondence should be addressed; E-Mail: jmr77@mju.ac.kr; \\ Tel.: +82-105-227-0419; Fax: +82-313-306-824.
}

Academic Editor: Neville Watson

Received: 7 August 2015 / Accepted: 20 October 2015 / Published: 23 October 2015

\begin{abstract}
This paper investigates several existing techniques for reducing high-availability seamless redundancy (HSR) unicast traffic in HSR networks for substation automation systems (SAS). HSR is a redundancy protocol for Ethernet networks that provides duplicate frames for separate physical paths with zero recovery time. This feature of HSR makes it very suited for real-time and mission-critical applications such as SAS systems. HSR is one of the redundancy protocols selected for SAS systems. However, the standard HSR protocol generates too much unnecessary redundant unicast traffic in connected-ring networks. This drawback degrades network performance and may cause congestion and delay. Several techniques have been proposed to reduce the redundant unicast traffic, resulting in the improvement of network performance in HSR networks. These HSR traffic reduction techniques are broadly classified into two categories based on their traffic reduction manner, including traffic filtering-based techniques and predefined path-based techniques. In this paper, we provide an overview and comparison of these HSR traffic reduction techniques found in the literature. The concepts, operational principles, network performance, advantages, and disadvantages of these techniques are investigated, summarized. We also provide a comparison of the traffic performance of these HSR traffic reduction techniques.
\end{abstract}

Keywords: high-availability seamless redundancy (HSR); HSR traffic reduction techniques; substation automation systems (SAS); smart grid communications 


\section{Introduction}

High-availability seamless redundancy (HSR) was standardized by the International Electrotechnical Commission (IEC) in IEC 62439-3 Clause 5 [1] and is one of the redundancy protocols selected for substation automation systems (SAS) in the IEC 61850 standard [2]. HSR is a redundancy protocol for Ethernet networks that provides zero switchover time in case of a network component failure. HSR is mainly applied for ring topologies. It is based on the duplication of every frame sent in a ring topology. Each copy of the frame is injected in a different direction of the ring. In the fault-free state of the network, the destination node receives two identical frames, passes the first frame to its upper layers, and discards the duplicate. In the case of failure of one network component such as a link failure or a node failure, only one frame is lost. The application on the destination node operates with the remaining frame undisturbed. Therefore, even in the case of a link or node failure, there is no communication interruption in the network. This feature of the HSR protocol makes it very useful for time-critical and mission-critical applications, such as SAS systems, automation networks, and military applications. The HSR principles and operations are described in detail in [1-7].

The major drawback of HSR is that it generates too much unnecessary redundant unicast traffic in HSR networks. This drawback results in the degradation of network performance and may cause congestion and delay. Several techniques have been proposed to reduce the unnecessary traffic in HSR networks. These HSR traffic reduction techniques can be classified into the following two categories: traffic filtering-based techniques and predefined path-based techniques. Traffic filtering-based techniques, such as quick removing (QR) [8-11], traffic control (TC) [12], pork locking (PL) [11,13,14], enhanced port locking (EPL) [15], and filtering HSR traffic (FHT) [16] filter unicast traffic for rings in HSR networks, and preventing the traffic from doubling and circulating in the rings. Predefined path-based techniques, such as optimal dual path (ODP) [17], dual virtual paths (DVP) $[11,18,19]$, and ring-based dual paths (RDP) [20] establish two separate paths, called dual paths, for each connection pair of terminal nodes or rings before transmitting traffic in HSR networks. The dual paths are then used to forward unicast frames from a source to a destination, instead of duplicating and forwarding frames to all parts of the networks, as in the standard HSR protocol.

In this paper, we provide a comparison of the current HSR traffic reduction techniques in the literature. The concepts, operational principles, performance, advantages, and disadvantages of these techniques are investigated, summarized, and compared.

The rest of this paper is organized as follows: in Section 2, we briefly introduce several existing redundancy protocols. Next, in Section 3, we introduce the HSR protocol and its issues. Section 4 describes traffic filtering-based techniques. Section 5 describes predefined path-based techniques. Finally, we provide our conclusions in Section 6.

\section{Existing Redundancy Protocols}

High seamless communication with fault tolerance is one of the key requirements for Ethernet-based, mission-critical, and real-time systems, such as substation automation systems, automation networks, and other industrial Ethernet networks. A fault-tolerant Ethernet eliminates single points of failure and therefore improves overall system availability. The Ethernet standardized by the Institute of Electrical 
and Electronics Engineers (IEEE) in IEEE 802.3 [21] is incapable of supporting a fault-tolerant network at all. A fault-tolerant Ethernet system is a common solution for addressing fault tolerance in Ethernet-based systems. Various fault-tolerant systems and redundancy protocols have been developed and standardized to provide fault tolerance and redundancy for Ethernet networks, such as fault-tolerant Ethernet (FTE) [22], scalable autonomous fault-tolerant Ethernet (SAFE) scheme [23], robust software-based fault-tolerant (RSAFE) scheme [24], spanning tree protocol (STP) [25], rapid spanning tree protocol (RSTP) [26], shortest path bridging (SPB) [27], transparent interconnection of lots of links (TRILL) [28-32], multiple MAC registration protocol (MMRP) [33], media redundancy protocol (MRP) [34], cross-network redundancy protocol (CRP) [35], beacon redundancy protocol (BRP) [36], parallel redundancy protocol (PRP) [1], high-availability seamless redundancy (HSR) [1] and others.

FTE [22] was created by Honeywell to provide rapid network redundancy. Each node is connected twice to a single local area network (LAN) through dual network interfaces. The driver and FTE-enabled components allow network communication to occur over an alternate route when the primary route fails. SAFE [23] is a software-based approach that provides network scalability and autonomous fault detection and recovery. SAFE adopts a heartbeat mechanism, which periodically sends heartbeat messages for fault detection. SAFE divides large-scale networks into several subnets by limiting the number of nodes in each subnet. This network can be easily configured as a star network to meet fault recovery time requirements. RSAFE [24] is an enhanced version of SAFE. It is also based on multiple subnets architecture and therefore incorporates most of the advantages of the SAFE scheme; however, the RSAFE scheme adopts novel algorithms to improve the failure switchover delay and eliminates the master nodes in each subnet to reduce the latency in inter-subnet communications.

STP [25] was standardized by the IEEE to provide redundancy in Ethernet networks. STP ensures that only one (active) path is used between any two nodes in an Ethernet LAN since logical loops are not allowed in Ethernet networks. STP can thus introduce network redundancy by having alternative paths that can be used by reconfiguring the network in case of a failure of a network link or switch. The main drawback of STP is the fairly slow network reconfiguration process, often taking about 30-50 s. This is far from satisfactory for most industrial applications. RSTP [26] has been developed as an enhancement to the original STP to provide faster network reconfiguration; however, RSTP still suffers switchover delay when failure occurs. SPB [27] and TRILL [28-32] were developed as alternatives to the spanning tree protocols. Both use the Intermedia System to Intermedia System (IS-IS) routing protocol instead of the spanning tree protocols and forward traffic via the shortest path between two nodes. SPB has been standardized by the IEEE as the next step in the evolution of the various spanning tree and registration protocols. SPB uses shortest path trees (SPTs) as alternatives to the spanning trees used by STP and RSTP. Shortest path trees guarantee that traffic is always forwarded via the shortest path between two nodes. SPB uses the IS-IS routing protocol to exchange information between nodes to calculate SPTs. SPB allows all paths to be active with multiple equal cost paths and provides much larger layer 2 topologies. It also allows for true shortest path forwarding in a mesh Ethernet network context utilizing multiple equal cost paths. SPB further provides fast connectivity restoration after failure. TRILL was standardized by the Internet Engineering Task Force (IETF) and is a layer 2 forwarding protocol that operates within one IEEE 802.1-compliant Ethernet broadcast domain. It replaces the spanning tree protocols by using IS-IS routing to distribute link state information and calculate the shortest paths through the network. TRILL is implemented by devices 
called routing bridges (RBridges). RBridges are compatible with and can incrementally replace previous IEEE 802.1 customer bridges. MMRP [33] is a layer 2 protocol for registering group MAC addresses (i.e., multicast) on multiple switches. It is a multiple registration protocol (MRP) application [33]. The purpose of MMRP is to allow multicast traffic in bridged LANs to be confined to the areas of the network where it is required.

Several redundancy protocols have been developed and standardized in the International Electrotechnical Commission (IEC) 62439 standard series to provide protocol-independent redundancy in substation automation networks, such as MRP [34], CRP [35], BRP [36], PRP [1], and HSR [1]; however, among these protocols, only two-PRP and HSR - are suitable for seamless communications. Both HSR and PRP are based upon the principle of providing duplicated frames for separate physical paths with a zero recovery time [1]. Unlike PRP, which requires dual redundant independent networks, HSR can be applied to a single network, in particular single rings and connected rings while simultaneously retaining its zero recovery time property.

\section{Background}

\subsection{HSR Concepts}

HSR follows the PRP principle of frame duplication over distinct paths [1]. Unlike PRP that requires a completely duplicated network topology, HSR uses a single network. The HSR protocol defines the following node types [1]:

- Doubly attached node for HSR (DANH): A DANH node is an HSR-capable switching end node that has two HSR ports sharing the same medium access control (MAC). These two ports form a single interface. The source DANH inserts the HSR tag into the frame received from upper layers, duplicates the frame into two frame copies and sends the two copies over both ports. The destination DANH receives - in the case of failure-free - two copies of the same frame, one from each port.

- Redundancy Box (RedBox): Singly attached nodes (SAN), such as servers, maintenance laptops or printers cannot be directly connected to an HSR ring since they have no the HSR forwarding capability and do not support the HSR tag. A RedBox is used to connect SANs to the HSR ring. RedBoxes forward the frames over the ring like DANH nodes and act as proxies for all SANs that access them.

- Quadruple port device (QuadBox): QuadBox nodes are used to connect HSR rings together. A QuadBox has four HSR ports that are divided into two pairs connected by an interlink; each pair shares the same MAC. QuadBoxes operate as HSR nodes towards both rings simultaneously.

HSR is mainly applied for ring topologies. The HSR protocol is used in single-ring and connected-ring networks.

\subsubsection{Single-Ring Networks}

In a single-ring HSR network, HSR uses the two HSR ports of each DANH node to interconnect all DANH nodes to the ring network, as shown in Figure 1. There is no QuadBox used in single-rings. 


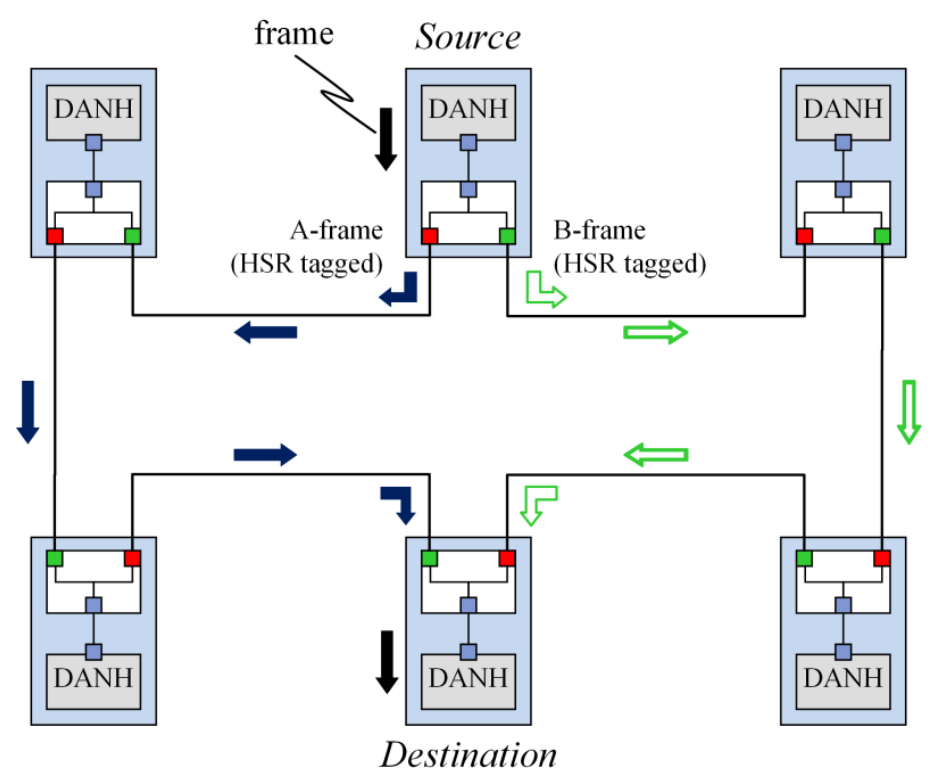

Figure 1. HSR example of single-ring topology for unicast traffic.

The source node sends two copies of the same frame simultaneously through each port in both directions in the ring. The two frame copies travel in opposite directions. If a DANH receives the frame, but is neither its source nor its only destination, the DANH forwards the frame to its other port, except if it already forwarded the same frame. The destination node of a unicast frame does not forward a frame for which it is the only destination. Frames forwarding in the ring carry the HSR tag inserted by the source node, which contains a sequence number. The doublet of source MAC address and sequence number uniquely identifies copies of the same frame. In the fault-free case of the network, the destination node receives two identical frames, passes the first frame to upper layers, and discards the duplicate. If a single fault on the ring occurs, only one of the two frames travelling on the two network paths through the ring will experience a communication interruption. The second frame will still reach the destination node.

\subsubsection{Connected-Ring Networks}

To allow more complex network topologies, HSR rings can be connected through the use of QuadBoxes. A QuadBox forwards frames over each ring, passing the frames to another ring without changes. Although one QuadBox is sufficient to conduct the traffic in a fault-free network, two QuadBox nodes are used to prevent a single point of failure [1]. Figure 2 shows an example of a connected-ring network that consists of four QuadBoxes used to connect four DANH rings.

\subsection{HSR Issues}

The forwarding rule of the standard HSR protocol is that nodes forward unicast frames from one port to other ports, unless they already sent the same frame in that direction. A node will not forward a frame that it injected into the ring [1]. 


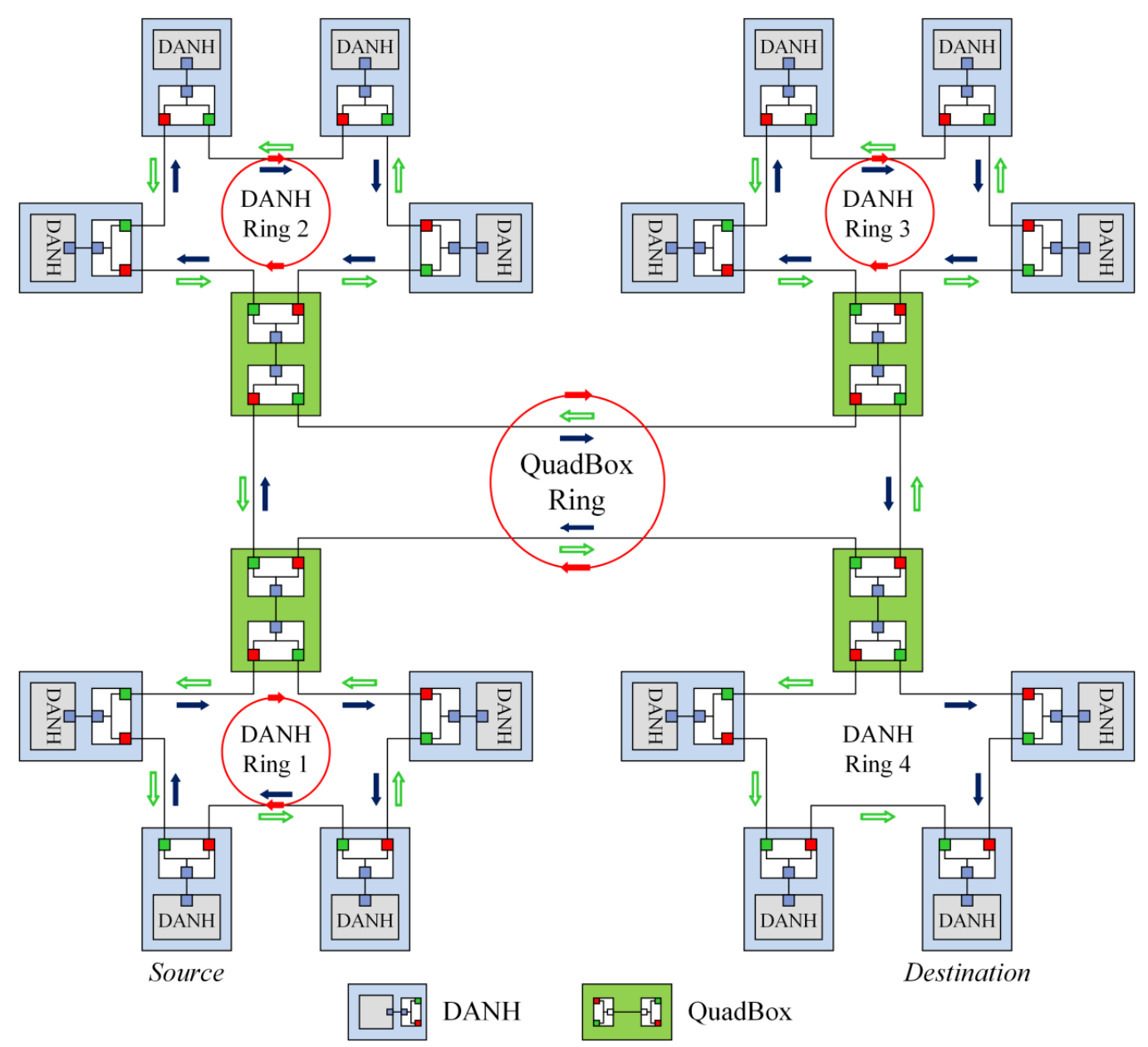

Figure 2. HSR example of connected-ring topology for unicast traffic.

HSR protocol has no issues with unicast frames in single-ring networks. However, HSR protocol has a serious drawback in connected-ring networks that is HSR generates too much unnecessary redundant unicast traffic. The drawback is caused by the following issues:

(1) Forwarding frames into all DANH rings, except the destination DANH ring.

(2) Forwarding frames into all QuadBox rings.

(3) Doubling and circulating frames in all the rings.

This drawback of HSR degrades the network performance and may cause network congestion and delay. Therefore, several techniques for reducing HSR unicast traffic have been proposed to solve the problem of HSR. These techniques can be classified into the following two categories: traffic filtering-based methods and predefined-paths based methods. Traffic filtering-based techniques prune unicast traffic for rings in HSR networks, and preventing the traffic from doubling and circulating in the rings. Predefined path-based techniques establish two separate paths for each connection pair of terminal nodes. These dual paths are then used to forward unicast frames between each connection pair of nodes, instead of duplicating and forwarding frames to all parts of the networks as in the standard HSR protocol.

We will describe these traffic filtering-based techniques and predefined path-based techniques in Sections 4 and 5, respectively. 


\section{Traffic Filtering-Based Techniques}

Traffic filtering-based techniques reduce the redundant traffic by solving some or all the issues related to HSR mentioned in Section 3. There are several traffic filtering-based techniques, as follows:

- $\quad$ Quick removing $(\mathrm{QR}): \mathrm{QR}$ removes duplicated and circulated traffic from rings.

- Traffic control (TC): TC also removes duplicated and circulated traffic from rings, like QR.

- $\quad$ Port locking (PL): PL prunes unicast traffic for DANH rings.

- Enhanced port locking (EPL): EPL is an enhanced version of the PL approach. The EPL approach prunes unicast traffic for both DANH and QuadBox rings.

- $\quad$ Filtering HSR traffic (FHT): FHT filters unicast traffic for both DANH and QuadBox rings and removes duplicated and circulated traffic in the rings.

Table 1 summarizes traffic filtering features of these techniques.

Table 1. Traffic filtering features of techniques.

\begin{tabular}{cccccc}
\hline Traffic Filtering Features & QR & TC & PL & EPL & FHT \\
\hline Filtering traffic for DANH rings & No & No & Yes & Yes & Yes \\
Filtering traffic for QuadBox rings & No & No & No & Yes & Yes \\
Removing duplicated and circulated traffic & Yes & Yes & No & No & Yes \\
\hline
\end{tabular}

Notes: Quick removing (QR), Traffic control (TC), Port locking (PL), Enhanced port locking (EPL), Filtering HSR traffic (FHT), Doubly attached node for HSR (DANH).

\subsection{Quick Removing $(Q R)$}

\subsubsection{QR Concepts}

The purpose of QR [8-11] is to remove duplicated and circulated frames from a ring when all the nodes have received one copy of the frame and begin to receive the second copy. QR is suited for ring or connected ring topologies and can be applied for any traffic, such as unicast, multicast and broadcast.

\subsubsection{QR Operations}

To understand the QR operation, we consider a single-ring network as shown in Figure 3. In that case, source node 2 has sent two copies A and B to all the ring nodes as the duplicated copies for a frame generated in the upper layer. Within four clocks, each of the frame copies, A and B, will pass each other at the link between nodes 5 and 6, and then travel towards nodes 5 and 6 , respectively. At this time, HSR nodes will take the following actions.

(a) If both frame copies are error-free: Nodes 5 and 6 have already received a copy of the frame. Node 5 has received copy $\mathrm{B}$ from the right side and then received copy A from the left side. The same is true for node 6; it has received copy A from the left side and then received copy B from the right side. In that case, nodes 1, 7 and 6 have received copy A and there is no need for copy B. The same is true for nodes 3, 4 and 5; they have received copy B and do not need to receive copy A. Therefore, node 6 will remove copy $\mathrm{B}$ from the ring and node 5 will remove copy A from the ring. 
(b) If a frame copy is corrupted as soon as it leaves the source node: We assume that copy A was corrupted when it left the source node 2 , moving towards node 1 . In this case, node 1 will remove copy A from the ring as soon as it receives copy A, and all the nodes in the rings, except the source node, will wait for copy B. Therefore, each node will receive a copy and forward it to the next node until it reaches the source node.

(c) If a frame copy is corrupted during transmission: We assume that copy A was corrupted during transmission between nodes 1 and 7. In that case, node 7 will remove copy A from the ring, and node 6 will not receive copy A. Therefore, node 6 will forward copy B as soon as it receives copy B from the right side. Node 7 will also forward copy B to node 1, but node 1 has already received copy A, so it will remove copy B from the ring.

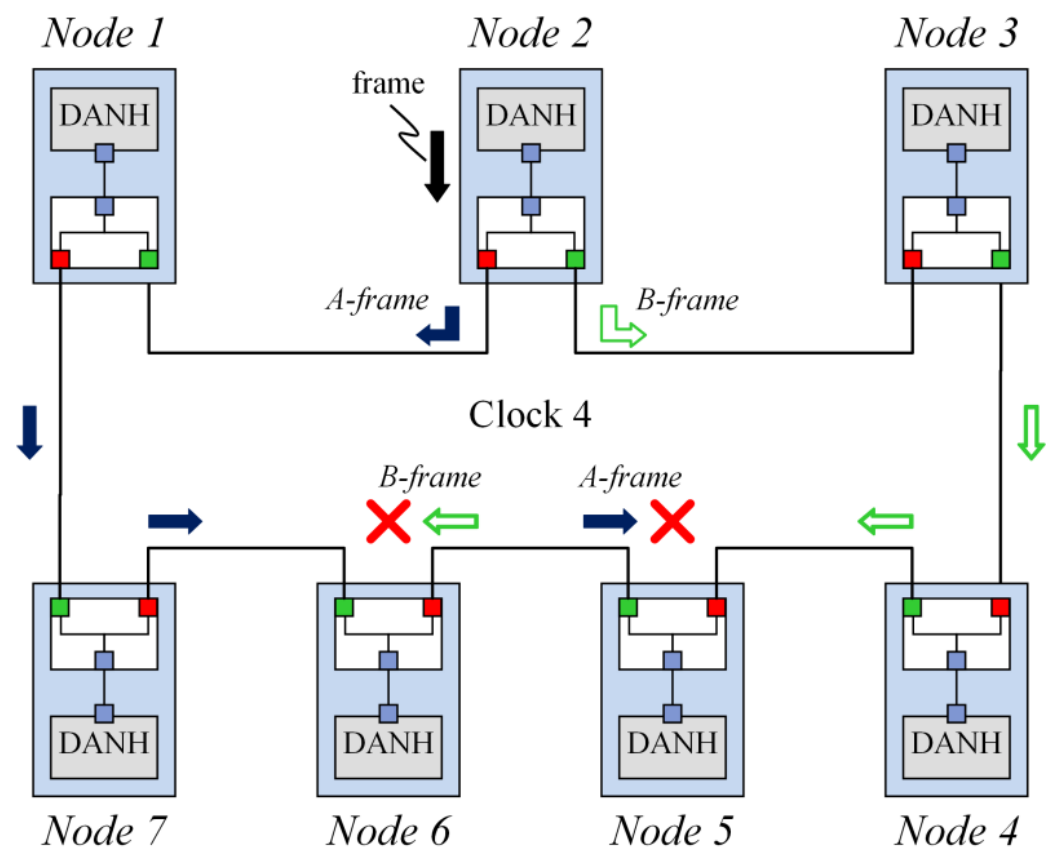

Figure 3. Removing duplicates in a single ring under the QR approach.

Figure 4 shows the process of removing duplicated and circulated frames under the QR approach in a sample network with six DANH rings and two QuadBox rings. In that case, the source node in DANH ring 1 sends unicast frames to the destination node in DANH ring 6. The QR approach removes duplicated and circulated traffic from all DANH and QuadBox rings. However, the traffic is forwarded into all rings of the network.

\subsubsection{Advantages and Disadvantages}

QR is the simplest approach to reduce redundant traffic in HSR networks. The QR approach is easy to implement. The QR approach can be applied for any traffic such as unicast, multicast and broadcast traffic, in any network topology.

However, the main disadvantage of $\mathrm{QR}$ is that it does not prune traffic for unused rings. In the $\mathrm{QR}$ approach, network unicast frames are forwarded into all rings of HSR networks, even DANH rings that do not contain the destination of the frames and QuadBox rings that are not used to deliver the unicast traffic from the source to the destination. 


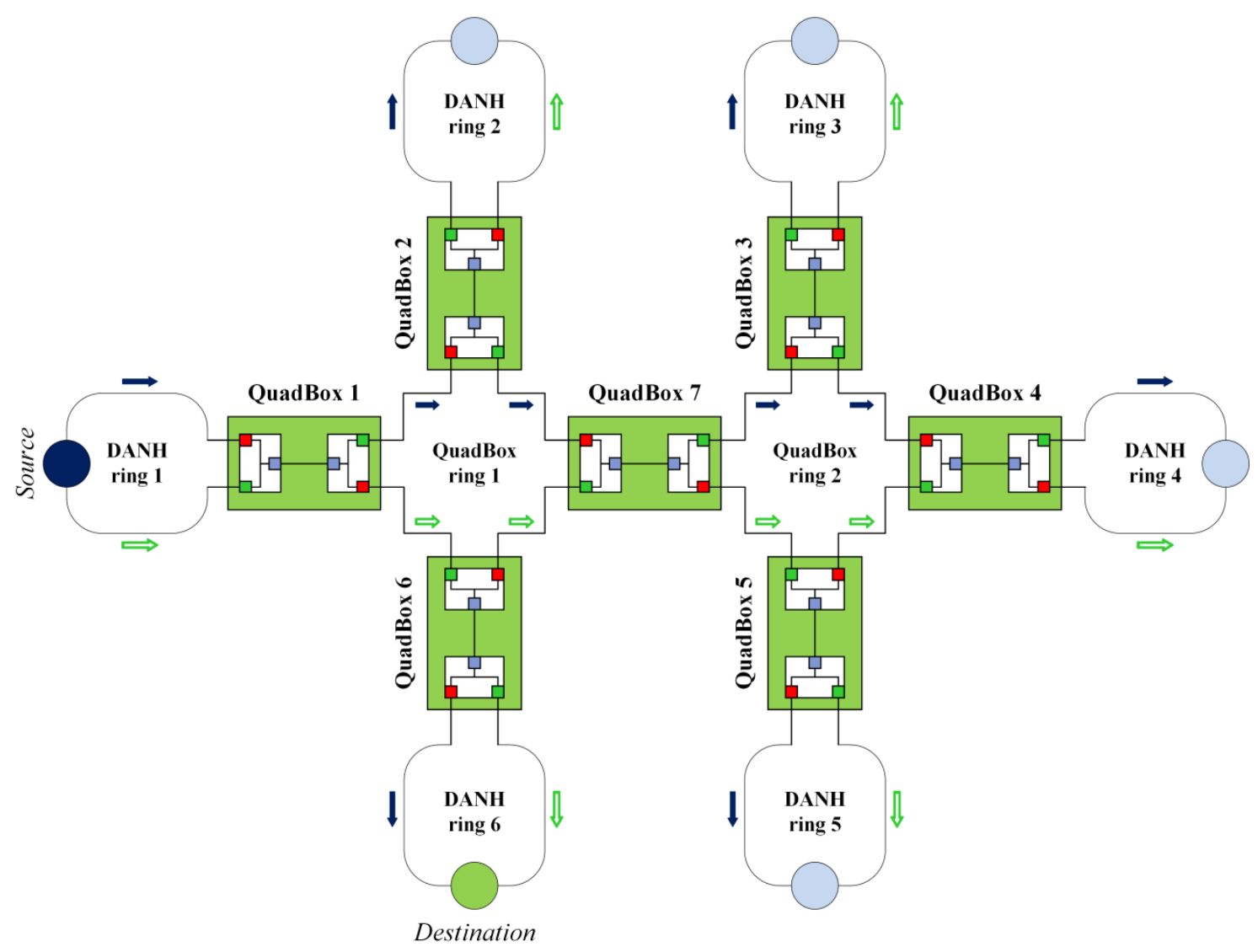

Figure 4. Removing duplicated and circulated frames under the QR.

\subsection{Traffic Control (TC)}

\subsubsection{TC Concepts}

Like QR, TC [12] proposed a simple method to remove duplicated and circulated frames from a ring. The TC approach is applied for terminal rings. The TC selects one of nodes within the ring as the traffic control node. The traffic control node will make decisions of discarding duplicated frames.

\subsubsection{TC Operations}

In the TC approach, end nodes are intelligent electronic devices (IEDs). IEDs connect to each other in a terminal ring. A source IED transmits two identical frames to a destination IED through both of its ports. First, the TC approach selects one of the IEDs within a ring as the traffic control IED (TCIED) of the ring. The TCIED of a ring has the function to keep information of all the IEDs in the ring through supervision frames. If the TCIED does not receive any duplicate of a frame which it has received before in a certain period, the TCIED will forward the frame.

Figure 5 shows the process of removing duplicated and circulated frames under the TC approach in a sample ring in the failure-free case. In that case, a source IED transmits two copies A and B of a frame through each port into the ring. The copy A that has first arrived at the TCIED is captured by the TCIED. The TCIED temporarily stores the copy and start a timer. If the TCIED receives the copy B of the frame in the timer interval, the TCIED will discard both copies A and B of the frame. 


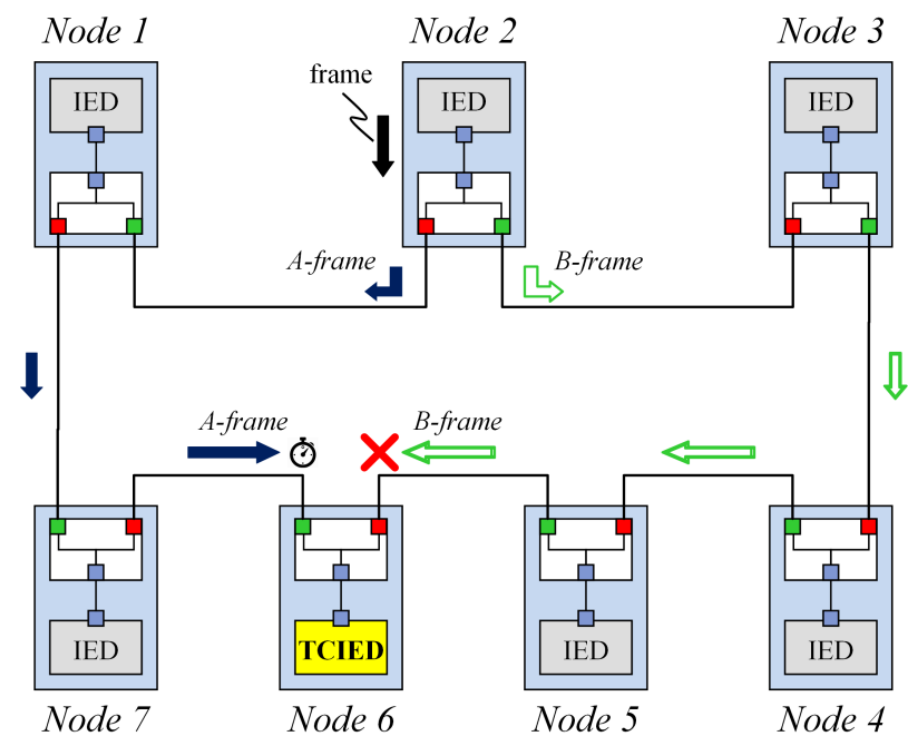

Figure 5. Forwarding frames in the failure-free case under the TC.

If there is a link failure occurred, for example the link between nodes 3 and 4 , the copy B cannot reach the TCIED. After the timer is expired, the TCIED forwards the copy A to next nodes, as shown in Figure 6.

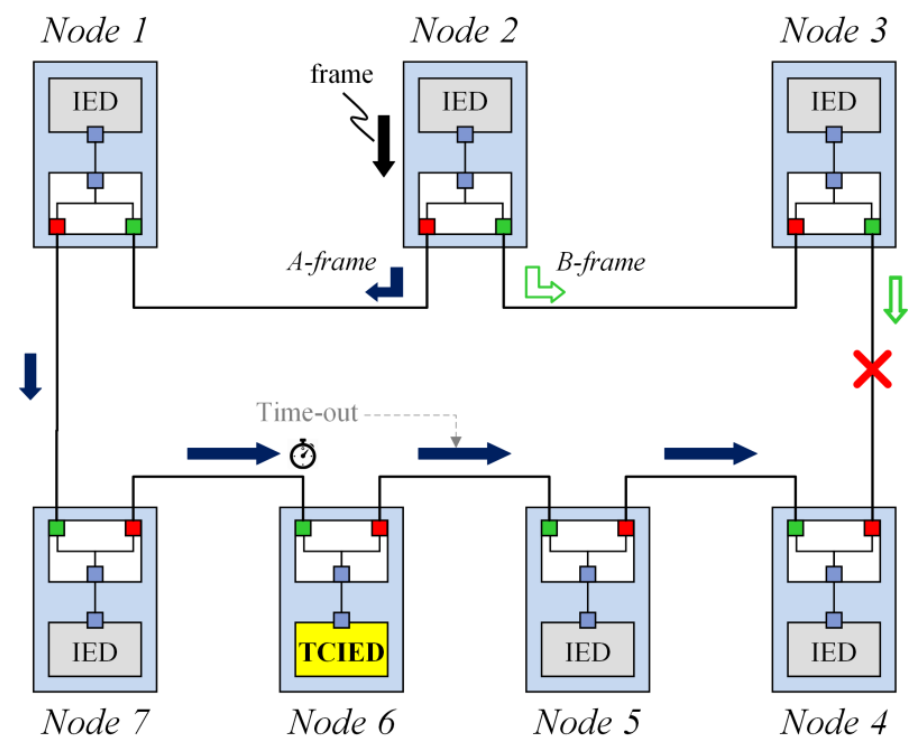

Figure 6. Forwarding frames in the failure case under the TC.

\subsubsection{Advantages and Disadvantages}

Like QR, the TC is a simple approach to reduce redundant traffic in HSR networks. The TC can be applied for any traffic such as unicast, multicast and broadcast in ring topologies.

However, the TC approach has some disadvantages. Like QR, the TC cannot prune traffic for unused rings. The TC allows network traffic to be forwarded into all rings of the networks. Unlike QR that removes circulated traffic from both DANH and QuadBox rings, the TC approach is applied for only DANH rings. In other words, unicast traffic is still duplicated and circulated in QuadBox rings. Additionally, the TC approach adds more delay to the traffic transmission. 


\subsection{Port Locking (PL)}

\subsubsection{PL Concepts}

PL $[11,13,14]$ reduces redundant unicast traffic in HSR connected-ring networks by filtering traffic for unused DANH rings. The PL approach makes the network learn gradually the location of the destination node without the network control messages. It then prunes DANH rings that do not contain the destination node by locking corresponding rings' entrance ports.

PL is only applied to QuadBoxes that connect to DANH rings. QuadBoxes that do not connect to any one of the DANH rings work as standard QuadBoxes. PL divides a QuadBox that connects to a DANH ring into two sides. One side, called DANH site, is connected to a DANH ring and the other side, called QuadBox side, is connected to a QuadBox ring. The DANH side of the QuadBox uses the PL approach while the QuadBox side continues working as a standard QuadBox. The reason behind dividing the QuadBox into two sides is to apply the PL approach to the DANH side only to prevent the QuadBox side from being locked. The destination node usually does not exist in the QuadBox ring. Therefore, frames sent from a QuadBox to any DANH ring without the destination node will be returned back the same QuadBox. If the PL approach is applied to the QuadBox side, then the QuadBox ring will be locked and the connection will be terminated.

When a source node sends frames to a destination node under the standard HSR protocol, each frame is tagged with an HSR tag, duplicated and circulated inside rings of the HSR network. If the destination node exists in a ring, it will take the frame without forwarding. If not, nodes in the network will forward the frame. When a QuadBox forwards a frame inside a DANH ring using one port and the frame is returned back to the QuadBox through the other port, it means that the destination of the frame does not exist in that ring. The PL will lock these ports to prevent them from sending the frames into the DANH ring.

\subsubsection{PL Operations}

The PL consists of two stages, the learning stage and working stage:

(a) Learning stage: In the learning stage, QuadBoxes check location of the destination node and perform port locking if needed. When the source node sends the first frame to the destination node, copies of the first frame are flooded into the entire network, as under the standard HSR protocol. QuadBoxes will check location of the destination of the frame, and then lock their DANH side's ports if DANH rings connecting to those ports do not contain the destination of the frame.

(b) Working stage: After the learning stage, all rings that do not contain the destination node are pruned. The working stage starts from the second frame. In the stage, frames are sent and received on paths through unlocked ports.

Figure 7 shows the process of filtering unicast traffic for DANH rings under the PL approach in the sample network. 


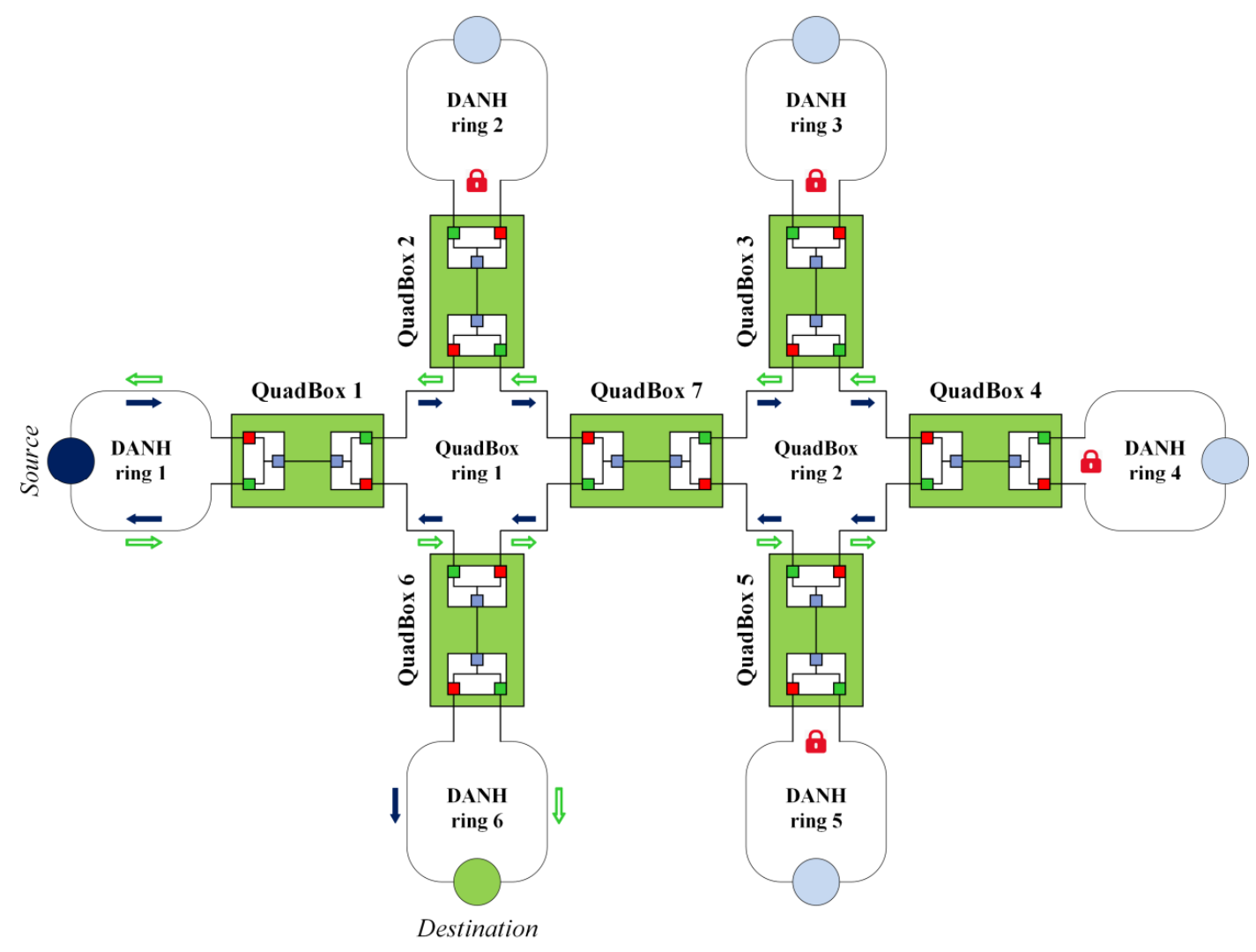

Figure 7. Filtering unicast traffic under the PL approach.

In that case, the source node in DANH ring 1 sends unicast frames to the destination node in DANH ring 6. In the leaning stage, QuadBoxes forward the first frame into DANH rings through their DANH side's ports. QuadBox nodes 2-5 then lock their DANH side's ports connected to DANH rings 2-5 respectively since they found that these DANH rings do not contain the destination of the frame. In the working stage, the frames are delivered from source node in DANH ring 1 to destination node in DANH ring 6 through all QuadBox rings, even unused QuadBox rings, such as QuadBox ring 2. Additionally, the frames are still doubled and circulated in all QuadBox rings.

\subsubsection{Advantages and Disadvantages}

The PL approach reduces redundant unicast traffic in HSR networks by filtering unicast traffic for unused DANH rings. Since the PL approach does not use control messages for filtering traffic, it avoids adding more control overhead in the HSR networks.

However, the PL approach has some disadvantages. The PL approach does not prune unicast traffic for unused QuadBox rings. Additionally, unicast frames are still duplicated and circulated in all QuadBox rings.

\subsection{Enhanced Port Locking (EPL)}

\subsubsection{EPL Concepts}

EPL [15] is an enhanced version of the PL approach. The EPL approach was proposed to improve the PL approach for unicast traffic performance in HSR connected-ring networks. The EPL works with 
the same locking concept as the PL. However, unlike the PL that prunes unicast traffic for only DANH rings, the EPL approach filters the unicast traffic not only for DANH rings, but also for QuadBox rings.

The EPL approach uses a parameter $\psi$ to trigger learning stages. Parameter $\psi$ is an integer number that represents the number of returned frames required to activate EPL effect. When a source node sends unicast frames to a destination node in an HSR network, the network will work as the standard HSR protocol at the first frame. From the second frame to the $\psi^{\text {th }}$ frame, the network will work under the PL effect to filter traffic for DANH rings. And from the $\left(\psi^{\text {th }}+1\right)$ frame, the network will work under the EPL effect to prune traffic for both DANH and QuadBox rings.

\subsubsection{EPL Operations}

The EPL approach works in three sequential stages:

- The first learning stage: DANH rings locking stage.

- The second learning stage: QuadBox rings locking stage.

- The working stage.

(a) The first learning stage: In the first learning stage, the network works as standard HSR during sending of the first frame, and all DANH rings that do not contain the destination of the frame will be pruned. At this stage, QuadBoxes that connect to the DANH rings will learn MAC addresses and build their PL locking tables. The PL locking table inside a QuadBox stores the MAC addresses of DANH nodes that do not belong to the QuadBox's DANH ring.

(b) The second learning stage: In the second learning stage, QuadBoxes that connect to a DANH ring without a destination node will lock their DANH side's ports and forward the unicast frames in the QuadBox ring. Conversely, QuadBoxes that connect to a DANH ring with a destination node will forward the unicast frames inside the DANH ring but will not forward the frames in the QuadBox ring. In the stage, QuadBoxes that connect to two QuadBox rings will build their EPL locking tables based on the same concepts used in the PL approach. The EPL locking table inside a QuadBox contains the MAC addresses of DANH nodes that do not belong to DANH rings that connect to the QuadBox's QuadBox ring. The second learning stage starts after the first learning stage has been completed and is triggered according to the parameter $\psi$. After receiving $\psi$ number of returned frames, the second learning stage will be triggered, and the EPL approach will decide to lock that interface by registering the destination MAC address in the EPL locking table. Putting a MAC address inside the EPL locking table means that the QuadBox will stop forwarding any frame tagged with that MAC address as a destination in that direction.

At the first frame, the network will work as the standard HSR protocol. From the second frame to the $\psi^{\text {th }}$ frame, the network will work under the PL effect. And from the $\left(\psi^{\text {th }}+1\right)$ frame, the network will work under the EPL effect.

Figure 8 demonstrates the two learning stages in the EPL approach. Figure 8a,b represent the first learning stage, whereas Figure 8c,d represent the second learning stage of a QuadBox that connects to a DANH ring without a destination node and a QuadBox that connects to a DANH ring with a destination node, respectively.

(c) Working stage: In the stage, QuadBoxes forward unicast frames based on PL and EPL locking tables. Therefore, only rings used to forward traffic will remain active while the unused rings will be pruned. 


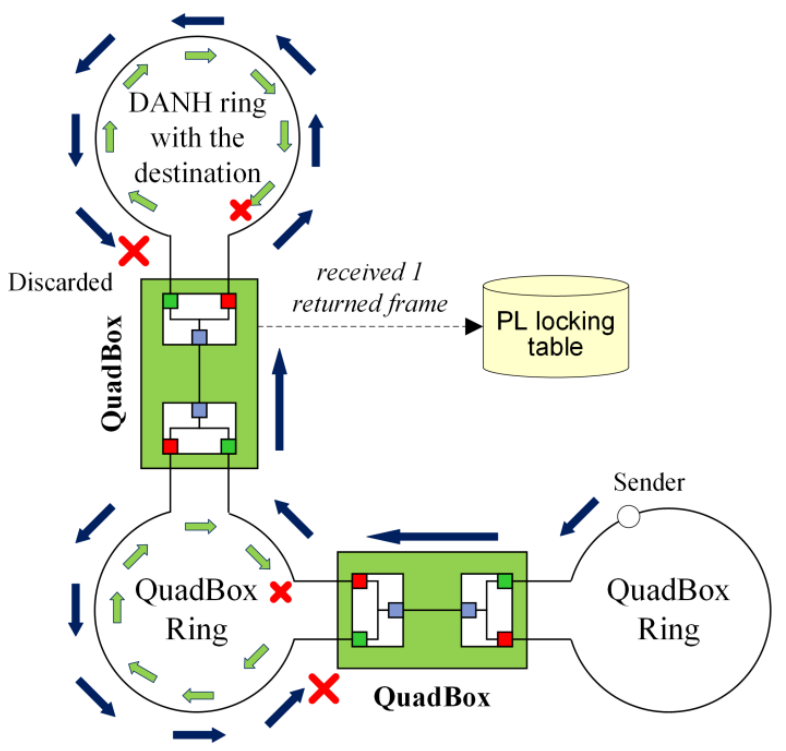

(a) The first learning stage at a QuadBox that connects to a DANH ring without the destination

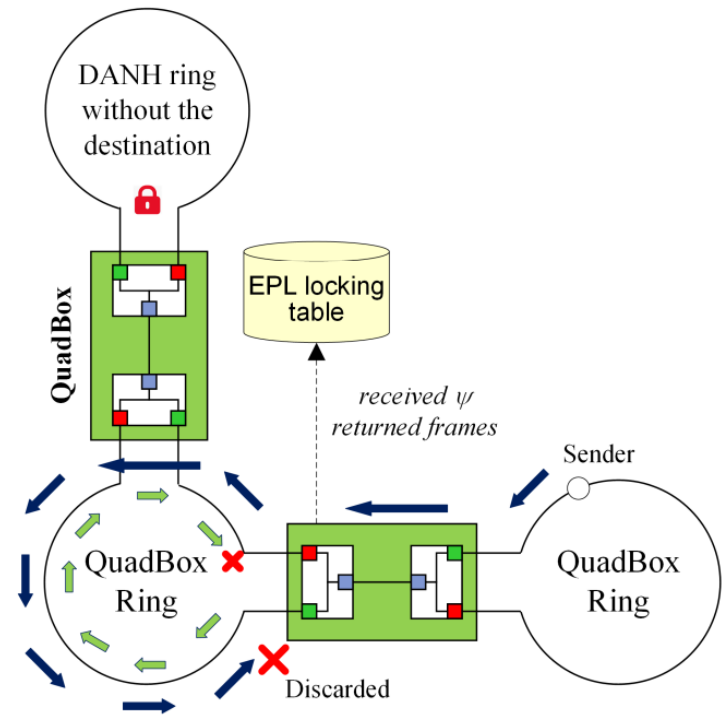

(c) The second learning stage at a QuadBox that connects to a DANH ring without the destination

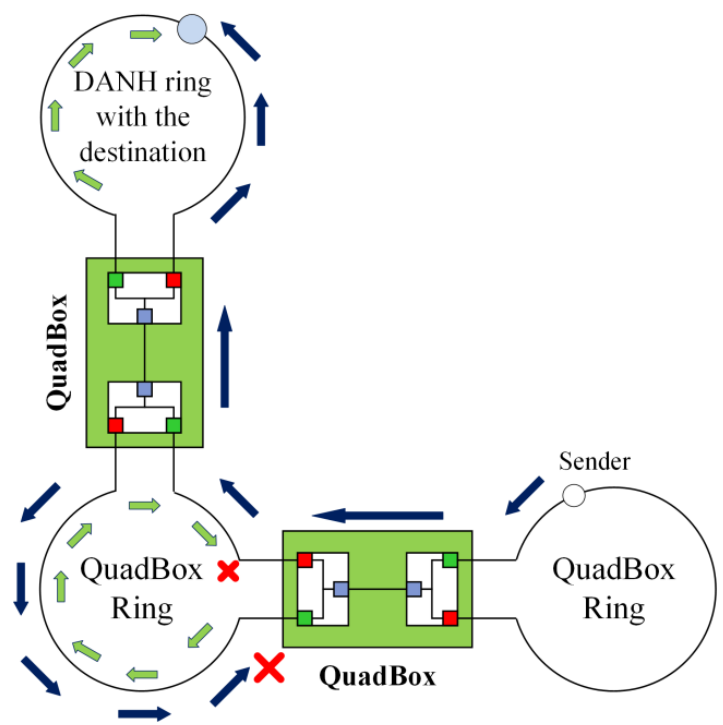

(b) The first learning stage at a QuadBox that connects to a DANH ring with the destination

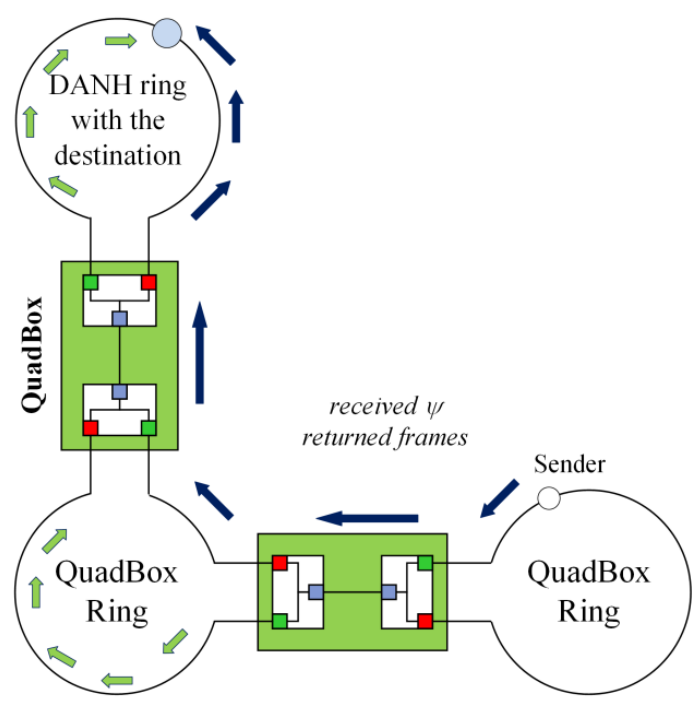

(d) The second learning stage at a QuadBox that connects to a DANH ring with the destination

Figure 8. The two learning stages in the EPL approach.

Figure 9 shows the process of filtering unicast traffic for rings under the EPL approach. In the first leaning stage, QuadBox nodes 2-6 build their PL locking table. QuadBoxes 2-5 will lock their DANH side's ports since the destination of the frame belongs to DANH ring 6. In the second leaning stage, QuadBox 7 builds its EPL locking table. QuadBox 7 will lock its ports that are connected to QuadBox ring 2 after it receives $\psi$ number of returned frames from the ring. After learning stages, QuadBox 2 blocked its DANH side's ports connected to DANH ring 2, and QuadBox 7 blocked its ports connected to QuadBox ring 2. In the working stage, the active ring path is DANH ring 1-QuadBox ring 1-DANH ring 6. In other words, the frames are delivered from source DANH ring 1 to destination DANH ring through only QuadBox rings 1. The frames are not forwarded into QuadBox ring 2, as in the PL approach. However, the unicast frames are still duplicated and circulated in active QuadBox rings (QuadBox ring 1 in this example) under the EPL approach. 


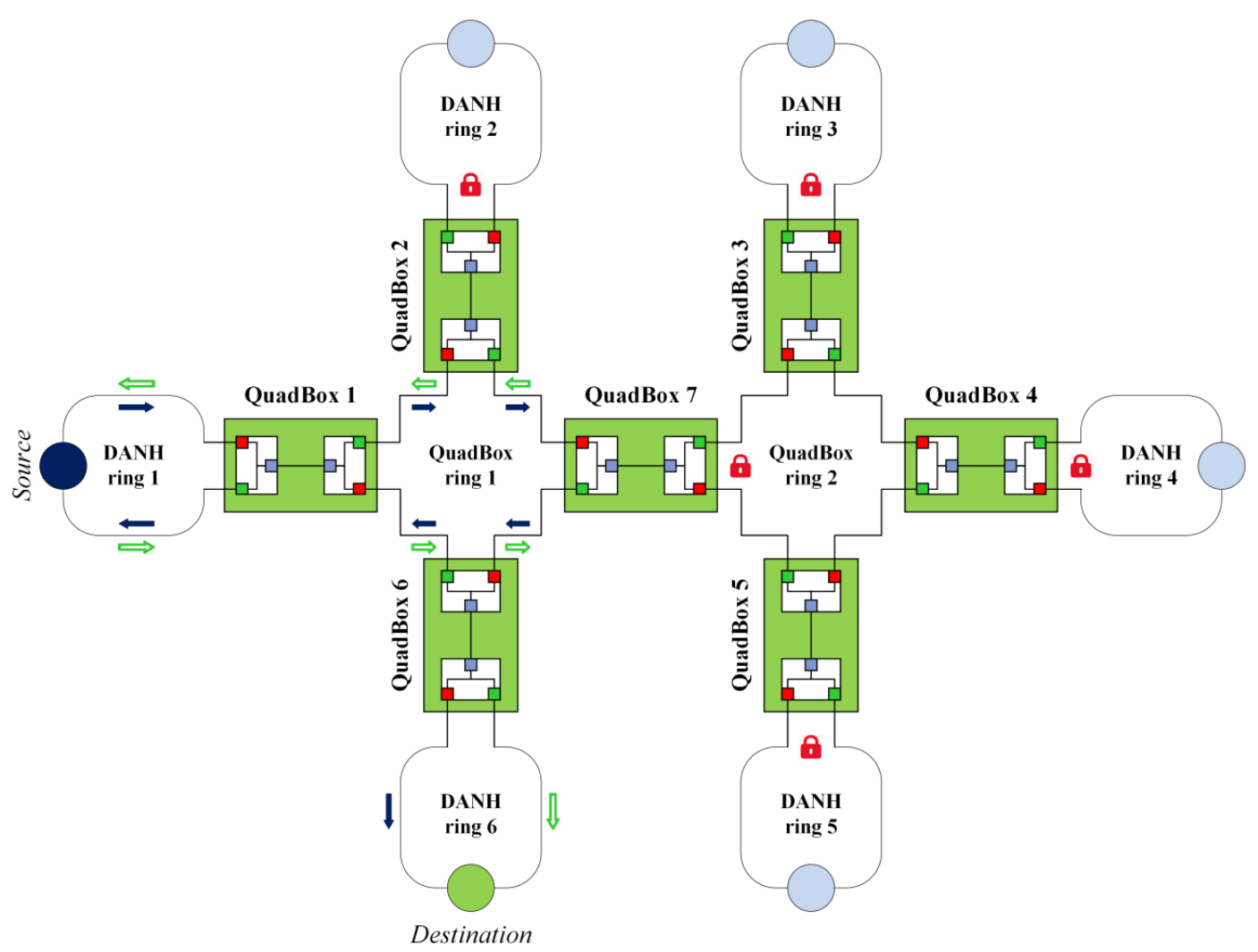

Figure 9. Filtering unicast traffic under the EPL approach.

\subsubsection{Advantages and Disadvantages}

As an enhanced version of the PL approach, EPL improves traffic performance in HSR networks compared with the PL approach. The EPL approach prunes unicast traffic not only for unused DANH rings, but also for unused QuadBox rings. Therefore, it provides better unicast traffic reduction than the PL approach. Additionally, like the PL approach, the EPL approach does not use control messages for pruning unicast traffic; thus there is no additional control overhead in the networks.

However, the EPL approach has some disadvantages. First, the EPL approach does not remove duplicated and circulated unicast frames from active QuadBox rings. Additionally, it is difficult to determine optimized value for the parameter $\psi$ that depends on the topology and size of HSR networks. Finally, since QuadBoxes learn MAC addresses of destination nodes that do not belong to their corresponding rings, the size of PL and EPL locking tables is large and directly proportional to the size of the network, resulting in high memory requirements.

\subsection{Filtering HSR Traffic (FHT)}

\subsubsection{FHT Concepts}

FHT [16] is an HSR traffic reduction technique that solves all HSR issues mentioned in Section 3. The FHT approach prunes unicast traffic for both unused DANH and QuadBox rings, and removes duplicated and circulated traffic from all active rings in HSR networks.

The FHT define two types of QuadBoxes: access QuadBoxes and trunk QuadBoxes. Access QuadBoxes are QuadBoxes that connect to at least one DANH ring, and trunk QuadBoxes are 
QuadBoxes that connect two QuadBox rings. To filter unicast traffic for unused DANH rings, each access QuadBox learns MAC addresses of DANH nodes that connect to its DANH ring and store these MAC addresses in a MAC1 table. To prune unicast traffic for unused QuadBox rings, each trunk QuadBox builds a MAC2 table that is a collection of MAC1 tables of access QuadBoxes that connect to its QuadBox rings.

To implement the functionality of filtering unicast traffic, the FHT approach defines two traffic filtering rules:

- Filtering rule 1: Filtering inbound traffic for DANH rings. Access QuadBoxes are used to filter inbound traffic for DANH rings based on their MAC1 table. An access QuadBox node forwards a unicast frame into its DANH ring if and only if its MAC1 table contains the destination MAC address of the frame.

- Filtering rule 2: Filtering outbound traffic for QuadBox rings. Trunk QuadBox nodes are used to filter outbound traffic for QuadBox rings based on their MAC2 table. A trunk QuadBox node forwards a unicast frame from its first QuadBox ring to its second QuadBox ring if and only if the first QuadBox ring's MAC2 table does not contain the destination MAC address.

Figure 10a shows the process of filtering inbound unicast traffic for a DANH ring at an access QuadBox node, and Figure 10b shows the process of filtering outbound unicast traffic for a QuadBox ring at a trunk QuadBox node.

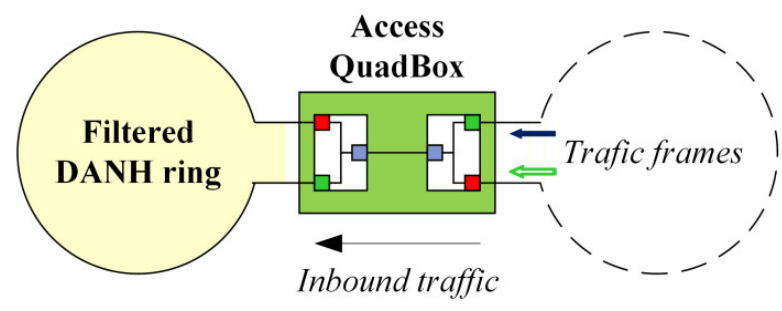

(a) Inbound traffic filtering for a DANH ring

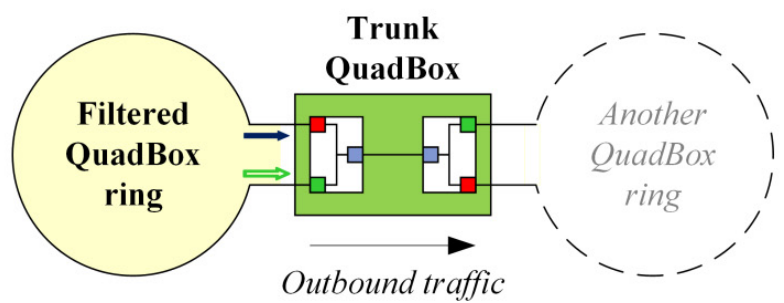

(b) Outbound traffic filtering for a QuadBox ring

Figure 10. The process of filtering unicast traffic.

Additionally, the FHT approach removes circulated traffic from active rings by defining a new traffic forwarding rule.

- Forwarding rule: QuadBoxes forward a unicast frame once, at most. When a QuadBox receives a unicast frame, it checks if the frame has previously been received and forwarded. If not, it forwards the frame. If so, it discards the frame. This new forwarding rule allows FHT to remove circulated traffic in rings.

\subsubsection{FHT Operations}

The FHT approach has two phases: learning phase and forwarding phase.

(a) Learning phase: In this phase, the FHT learns and builds MAC tables using control messages.

- Building MAC1 table: The MAC1 table is built at access QuadBoxes. Each QuadBox periodically sends a Hello message over all its ports. When a DANH node receives a Hello message, it replies by sending an ACK message, and forwards the Hello message to other 
DANH nodes. Each access QuadBox builds its MAC1 table based on ACK messages received from DANH nodes in its DANH ring. Based on the MAC1 table, an access QuadBox will not forward frames into its DANH ring if its MAC1 table does not contain the destination MAC address.

- Building MAC2 table: The MAC2 table is built at trunk QuadBoxes. Once access QuadBoxes have built their MAC1 tables, they send MAC messages that contain all the MAC addresses of their MAC1 table to their QuadBox ring. Trunk QuadBoxes connected to the QuadBox ring receive the MAC messages and update their MAC2 tables. Based on the MAC2 table, a trunk QuadBox will not forward a unicast frame from its first QuadBox ring to its second QuadBox ring if the first QuadBox ring's MAC2 table contains the destination MAC address.

(b) Forwarding phase: In the phase, QuadBoxes forward unicast frames based on MAC tables. The MAC1 table is used to prune the unicast frames for unused DANH rings, whereas the MAC2 table is used to filter the traffic for unused QuadBox rings. Additionally, based on the new traffic forwarding rule, the unicast frames are not doubled and circulated in the active rings.

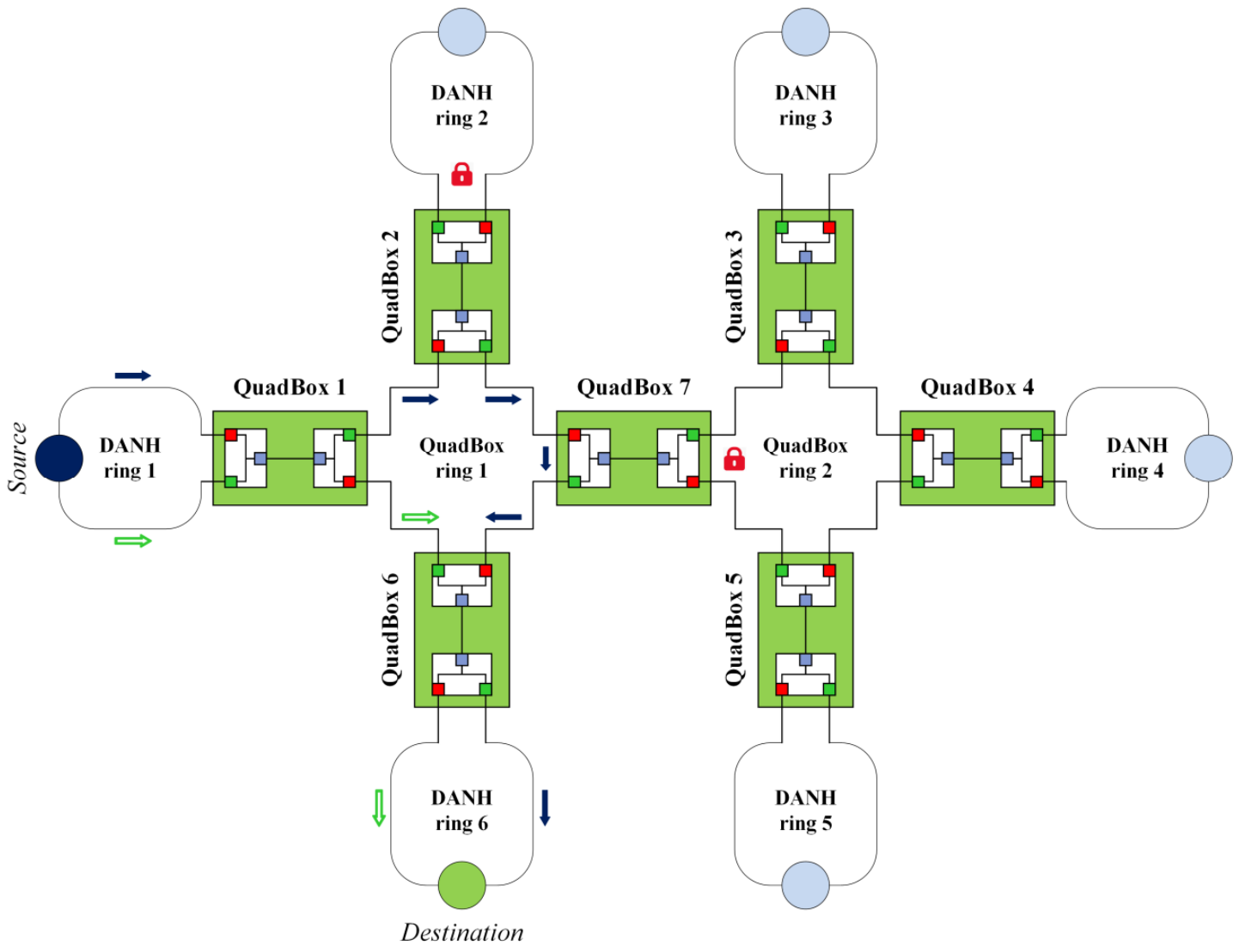

Figure 11. Filtering unicast traffic under the FHT.

Figure 11 shows the process of forwarding a unicast frame from a source to a destination under FHT. The source node sends two copies of the frame simultaneously through each port in both directions in DANH ring 1. Access QuadBox 1 passes the frame to its QuadBox ring 1. When receiving the frame from QuadBox ring 1, access QuadBox 2 looks up its MAC1 tables and does not forward the frame to its DANH ring 2, since its MAC1 table does not contain the destination MAC 
address of the frame. Access QuadBox 2 sends the frame over another trunk port to trunk QuadBox 7. Trunk QuadBox 7 looks up its MAC2 table and then does not forward the frame from its QuadBox ring 1 to its QuadBox ring 2, since its QuadBox ring 1's MAC2 table contains the destination MAC address. Finally, access QuadBox 6 forwards the frame into its DANH ring 6 because its MAC1 table contains the destination MAC address of the frame. The destination node receives two copies of the frame from both directions of DANH ring 6, passes the first frame to its upper layers, and discards the duplicate. According to the new forwarding rule for QuadBox nodes, the frame is not duplicated and circulated in active QuadBox rings.

\subsubsection{Advantages and Disadvantages}

The FHT approach prunes unicast traffic for not only unused DANH rings but also unused QuadBox rings. Additionally, it also removes duplicated and circulated traffic from the active rings. These features make the FHT approach become one of the most efficient HSR traffic reduction techniques. Since FHT's MAC tables contain only MAC addresses of DANH nodes that belong to DANH rings, the FHT approach requires low memory requirements compared with the PL and EPL approaches. The main drawback of the FHT approach is that it uses control messages to learn MAC addresses and build MAC tables. This results in additional control overhead in HSR networks.

\subsection{Comparison}

We described five traffic filtering-based techniques for reducing HSR unicast traffic. QR and TC approaches remove duplicated and circulated unicast traffic from rings in HSR networks. TC removes circulated traffic from only DANH rings, while the QR removes circulated traffic from both DANH and QuadBox rings. However, both $\mathrm{QR}$ and TC do not prune unicast traffic for unused rings. The PL prunes unicast traffic for unused DANH rings, while the EPL prunes the traffic for both unused DANH and QuadBox rings. However, the PL and EPL do not remove circulated unicast traffic from active rings. The FHT filters unicast traffic for both unused DANH and QuadBox rings, and also remove duplicated and circulated traffic from active rings. Characteristics of these techniques are summarized in Table 2.

Table 2. Characteristics of traffic filtering-based techniques.

\begin{tabular}{cccccc}
\hline Traffic Filtering Features & QR & TC & PL & EPL & FHT \\
\hline Filtering traffic for DANH rings & No & No & Yes & Yes & Yes \\
Filtering traffic for QuadBox rings & No & No & No & Yes & Yes \\
Removing duplicated and circulated traffic & Yes & Yes & No & No & Yes \\
Learning MAC addresses & No & No & Yes & Yes & Yes \\
Using control messages & No & No & No & No & Yes \\
Filtered traffic & Any & Any & Unicast & Unicast & Unicast \\
Network topology & Ring & Ring & Ring & Ring & Ring \\
\hline
\end{tabular}

To compare traffic performance of these techniques, we conducted several simulations using the OMNeT++ simulation tool [37]. We consider a sample network consisting of three QuadBox rings and eight DANH rings; each DANH ring has four DANH nodes, as shown in Figure 12. Two QuadBoxes 
are used to prevent a single point of failure in the network. In these simulations, a source node in DANH ring 1 sends unicast frames to a destination node in DANH ring 5.

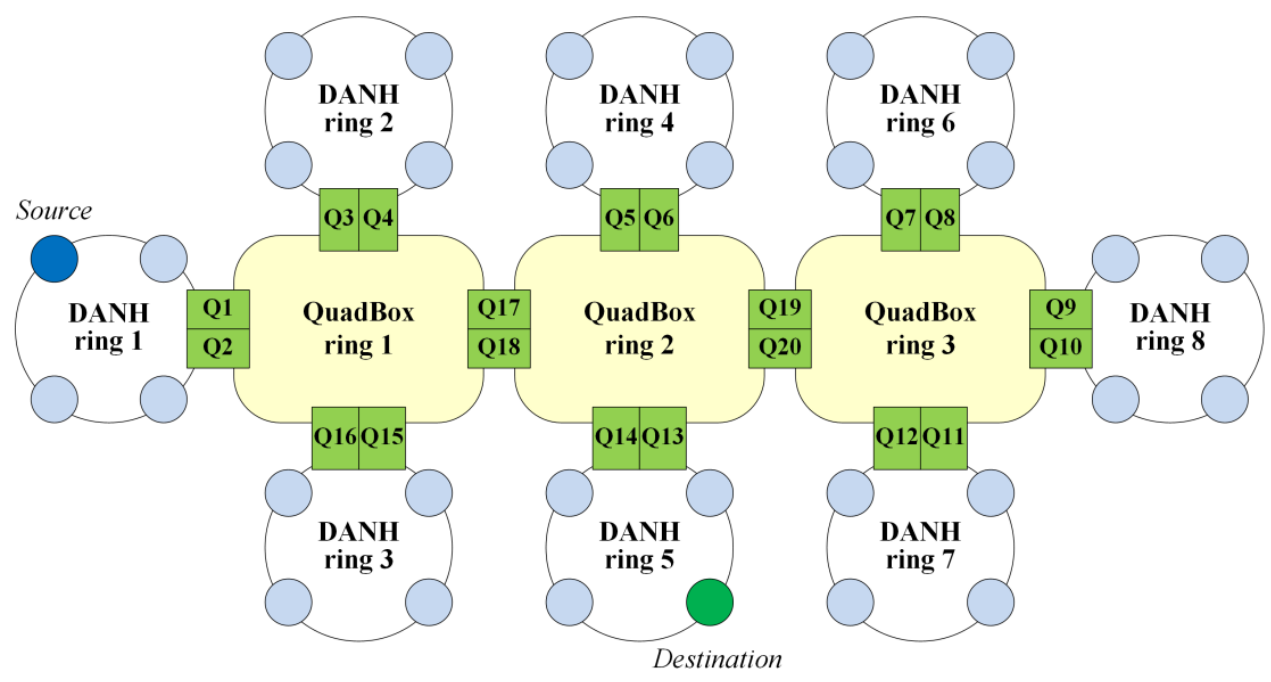

Figure 12. The simulation network.

Table 3 shows the number of network unicast frames recorded from the simulations under the standard HSR protocol, the QR approach, the PL approach, the EPL approach, and the FHT approach.

Table 3. The number of network traffic frames of traffic reduction techniques.

\begin{tabular}{cccccccccc}
\hline Number of Sent Frames & HSR & QR & TC & PL & EPL & FHT & ODP & DVP & RDP \\
\hline 10 & 1390 & 820 & 960 & 732 & 620 & 220 & 220 & 220 & 220 \\
20 & 2780 & 1640 & 1920 & 1392 & 1120 & 440 & 440 & 440 & 440 \\
30 & 4170 & 2460 & 2880 & 2052 & 1620 & 660 & 660 & 660 & 660 \\
40 & 5560 & 3280 & 3840 & 2712 & 2120 & 880 & 880 & 880 & 880 \\
50 & 6950 & 4100 & 4800 & 3372 & 2620 & 1100 & 1100 & 1100 & 1100 \\
60 & 8340 & 4920 & 5760 & 4032 & 3120 & 1320 & 1320 & 1320 & 1320 \\
70 & 9730 & 5740 & 6720 & 4692 & 3620 & 1540 & 1540 & 1540 & 1540 \\
80 & 11,120 & 6560 & 7680 & 5352 & 4120 & 1760 & 1760 & 1760 & 1760 \\
90 & 12,510 & 7380 & 8640 & 6012 & 4620 & 1980 & 1980 & 1980 & 1980 \\
\hline
\end{tabular}

Figure 13 shows the comparison of traffic performance of these techniques. The simulation results show that, in the sample network, the TC and QR approaches reduce network unicast traffic by $31 \%$ and $41 \%$, respectively, compared to the standard HSR protocol. The PL approach has better traffic performance than the QR approach; it reduces network unicast traffic by 51\% compared to standard HSR protocol and by about 17\% compared to the QR approach. The EPL improves traffic performance for the PL approach; it reduces network unicast traffic by $61 \%$ compared to standard HSR protocol and by about $21 \%$ compared to the PL approach. FHT provides the best traffic performance. For our sample network, the FHT approach reduces network traffic by $84 \%$ compared to standard HSR protocol and by about $57 \%$ compared to the EPL approach. 


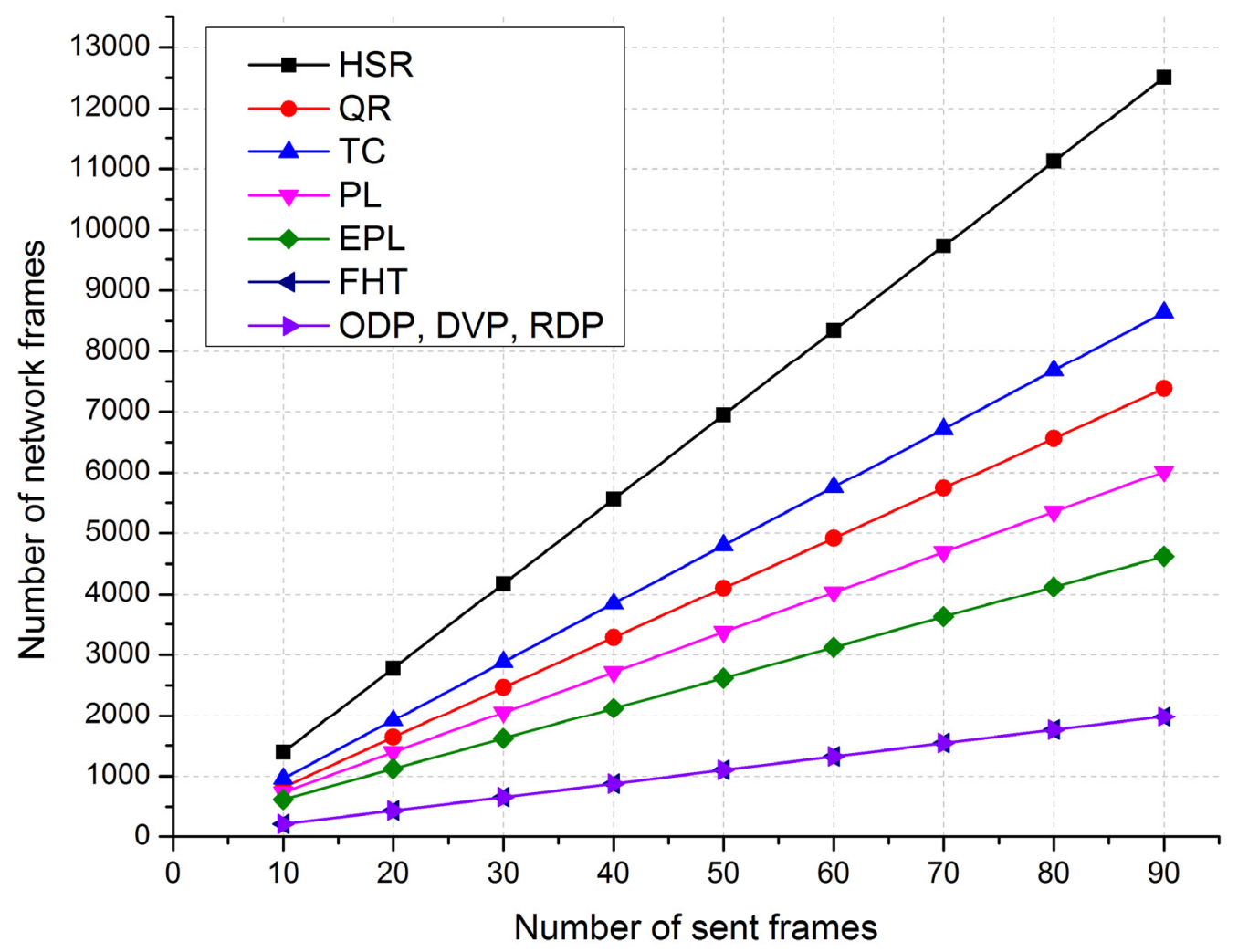

Figure 13. A comparison of the traffic performance of traffic reduction techniques.

\section{Predefined Path-Based Techniques}

Predefined path-based techniques reduce redundant traffic in HSR networks by forwarding unicast traffic through two separate predefined paths instead of duplicating and flooding the traffic as in the standard HSR protocol. There are several predefined path-based techniques, as follows:

- Optimal Dual Paths (ODP): ODPs establish dual paths that have optimal link metrics and no common nodes based on network link information.

- Dual Virtual Paths (DVPs): DVP establish dual paths for each connection pair of terminal nodes by sending and receiving control messages.

- $\quad$ Ring-based Dual Paths (RDP): RDP establish dual paths by sending and receiving control messages for each connection pair of terminal rings (DANH rings) instead of terminal nodes.

\subsection{Optimal Dual Paths (ODP)}

\subsubsection{ODP Concepts}

ODP [17] establishes dual paths for each connection pair of terminal nodes, such as DANH nodes, RedBox nodes, in HSR networks. The dual paths are determined based on the following criteria:

- The dual paths have optimal link metrics.

- The dual paths have no common nodes.

Each terminal node automatically establishes dual paths with the other terminal nodes in a network. To determine the dual paths with optimal metrics, each terminal node has to know the metric values 
for all of the network links. Therefore, the ODP approach builds a link metric table, which contains metrics of all the network links. Based on that table, the ODP approach searches all the available paths for each connection pair, sorts them in ascending order of path metrics, and determines dual paths that satisfy the above conditions. After selecting the optimal dual paths, each terminal node informs intermediate QuadBox nodes that are located in the dual paths about the selected dual paths that are passing through that QuadBox nodes. The QuadBox nodes save the path information, and they then forward received frames of that connection pair to a proper output port according to the previously learned information. In this case, the QuadBox node does not duplicate and flood the frames, as in the standard HSR protocol.

\subsubsection{ODP Operations}

The establishment process for the dual paths consists of three phases. In the first phase, nodes exchange their link information. In the second phase, each terminal node builds its link metric table. In the third phase, each terminal node determines the dual paths with the other terminal nodes based on the link metric table, and then informs the intermediate QuadBoxes in order to complete the establishment of the dual paths:

(a) Exchanging link metrics: In this phase, nodes exchange their link metrics so that each node knows link metrics of the entire network. This is accomplished by broadcasting a link metric (Met) message. However, to reduce the size and total number of exchanged Met messages, the node with the lowest node identifier (ID) broadcasts its link metrics to the other nodes, and the adjacent nodes that connect directly to it and have a higher ID do not broadcast the metrics of the shared links. However, these nodes will broadcast for other links they have.

(b) Building link metric table: Based on received Met messages, each terminal node builds a link metric table that contains the metrics of all links of the network. Ideally, all of the terminal nodes will have the same link metric table.

(c) Establishing dual paths: Based on the link metric table, each terminal node establishes dual paths with each corresponding terminal node. To do so, the following procedure is performed.

- Searching all available paths: The ODP approach searches the link metric table of a terminal node for all the available paths from that node to all the other terminal nodes in the network. The available paths are then added to a list called the available paths list. The paths list also has the metrics for each path.

- Sorting the searched paths list: The available paths are sorted in ascending order of the path metrics. The path with the lowest metrics is at the beginning of the list, next are the paths with higher metrics values, and the path with the highest metrics is at the end of the list.

- Selecting dual optimal paths: Based on the sorted paths list, the ODP approach determines dual paths for each connection pair of terminal nodes. The paths determination process depends on selecting paths with optimal links metrics that have no common nodes between them, except for the source and the destination nodes. To establish dual paths for a connection pair, the ODP approach first sets the first path in the sorted paths list as the first candidate path. Then, it compares the first candidate path with the other paths listed after it in the 
sorted paths list. If there is a path that has no common node with the first candidate path, then the optimal dual paths are found. If no available path is found, the algorithm selects and sets the next path of the current first candidate path as the new first candidate path and conducts a comparison. The iteration ends once a path that has no common node with the first candidate path is found.

- Confirming the selection: After selecting the dual paths for each connection pair, the terminal nodes send messages to inform the selected paths. QuadBoxes build their forwarding table based on the messages.

Figure 14 shows the process of forwarding unicast frames from source node 1 to destination node 10 under the ODP approach. For the connection pair of DANH nodes 1 and 10, the ODP approach has established dual paths, including the first path of DANH 2-DANH 3-DANH 4-QuadBox 2-QuadBox 3-QuadBox 4-QuadBox 13-QuadBox 5-DANH 9, and the second path of QuadBox 1-QuadBox 12-QuadBox 11-QuadBox 14-QuadBox 10-QuadBox 9-QuadBox 8-QuadBox 7-QuadBox 6-DANH 12-DANH 11. Each of these paths transfers only one frame copy from source node 1 to destination node 10, without duplication and flooding, because QuadBox nodes in between these paths forward the frame based on their forwarding table.

\subsubsection{Advantages and Disadvantages}

ODP significantly reduces redundant unicast traffic compared with the standard HSR protocol by forwarding the unicast traffic through two predefined paths instead of duplicating and flooding in overall network. Additionally, the dual paths have optimal link metrics, resulting in the improvement of quality of service in the network. The main drawback of ODP is that it has to build and maintain the link information table, resulting in additional control overhead in the network.

\subsection{Dual Virtual Paths (DVP)}

\subsubsection{DVP Concepts}

Like ODP, DVP [11,18,19] also establishes two predefined paths for each connection pair of terminal nodes in a network. However, unlike ODP that discovers the dual paths based on the network's link information, the DVP approach discovers the dual paths by sending and receiving control messages such as path selection and path confirmation messages. These dual paths will be automatically established in a DVP setup process. Such a process simply makes all of the intermediate QuadBox nodes "smart" after a learning task. The QuadBox nodes then understand how to forward a unicast frame to a proper destination through only one of their ports, instead of duplicating and flooding the frame through all ports, except the port on which the frame was received, as in standard HSR protocol. 


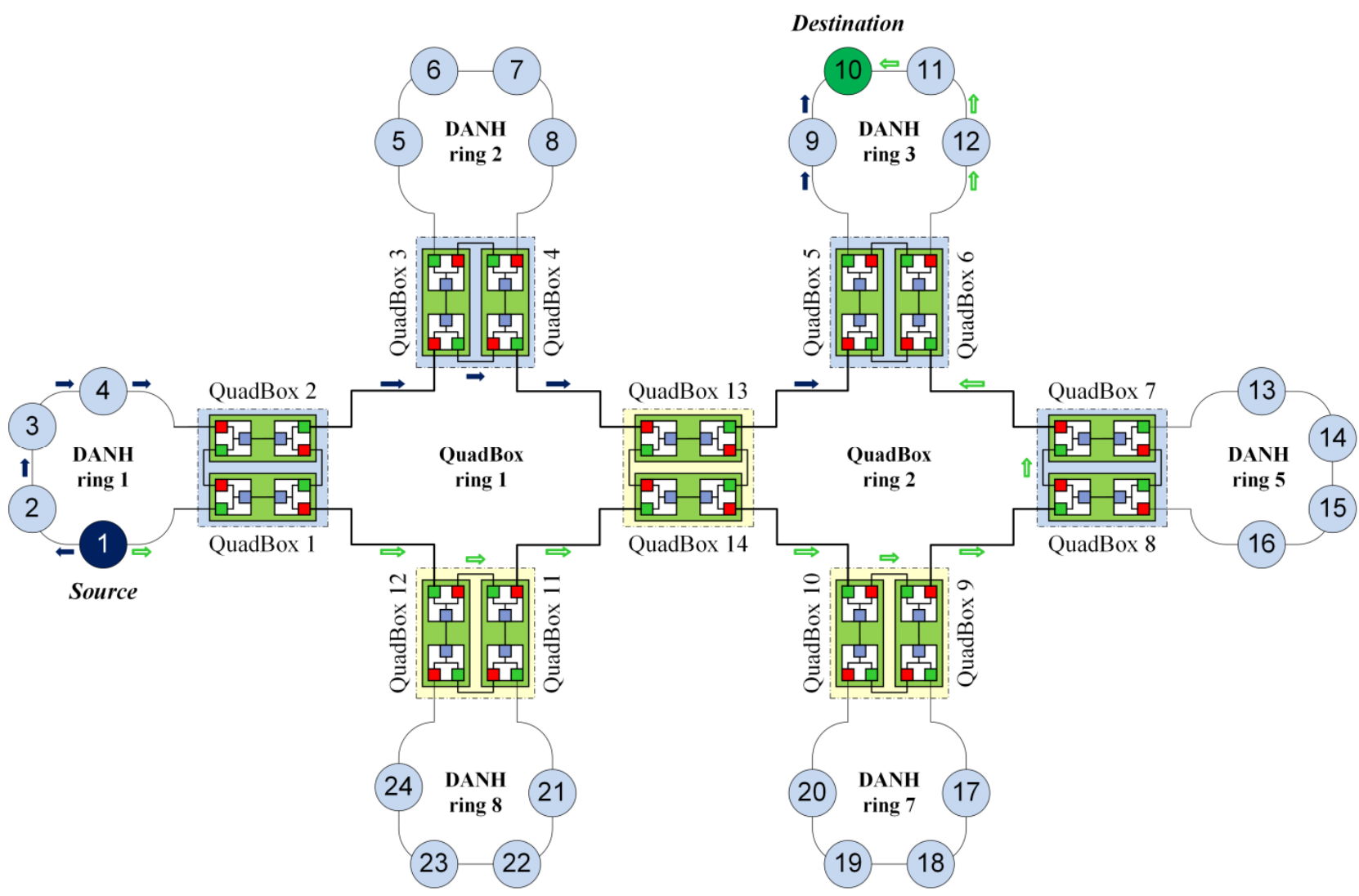

Figure 14. Forwarding unicast traffic under the ODP approach.

\subsubsection{DVP Operations}

The dual paths establishment of DVP consists of three phase, including announcement phase, path establishment phase, and final phase.

(a) Announcement phase: In the first phase, each node builds a neighbor $(\mathrm{Ne})$ table that contains MAC addresses of all terminal nodes. To build the Ne table, each terminal node announces itself by broadcasting an announcement (Ann) message in a network. Based on the Ann messages, all nodes in the network build the Ne table.

(b) Path establishment phase: In this phase, all the terminal nodes establish dual virtual paths with each other and use them for point-to-point communications. This task will be done by teaching QuadBox nodes how to forward the received frames to their destinations without duplicating and forwarding them randomly. This mission is accomplished by sending a path selection (PaS) message from each terminal node to each node listed in its Ne table. In other words, the terminal nodes of each connection pair send a PaS message to each other.

When a QuadBox receives a PaS message, it saves information of the message including the sequence number, source MAC address and destination MAC address into a pre-path (PrP) table, and then appends its identification (ID) tag, duplicates the tagged PaS message and sends them out. When a terminal node receives a PaS message that does not belong to its connection pairs, the terminal node forwards the message or deletes it if it has been forwarded before in the same direction. Each terminal node receives two copies of each $\mathrm{PaS}$ message sent to it, one from each direction, then builds or updates its $\mathrm{PrP}$ table, and finally replies with a path confirmed $(\mathrm{PaC})$ message. Each copy of the $\mathrm{PaC}$ 
message will travel through one of established dual virtual paths, and during this journey, each QuadBox will learn which port leads to the destination terminal node of that connection pair. Thus, all of the QuadBox nodes that the PaC message have been passed through become ready to forward any frame in both directions of that connection pair. The PrP tables will also be updated after this step. Eventually, after the building process of the PrP tables of all the nodes is complete, each QuadBox node will deduce another table from its PrP table to use for forwarding the received frames in both directions. This table is called the final path (FP) table. This FP table shows the input and output ports for each path.

(c) Final phase: After completing the building process of the FP tables, the DVP approach becomes ready to use, and any data frame that needs to be sent to a destination node in a connection pair will be duplicated at its source node, and then each copy will be sent into a virtual path that has been established earlier with that destination terminal node. Each QuadBox will forward the received unicast frames to their destination terminal nodes by using its FP table.

Figure 15 shows the process of forwarding unicast frames from source node 1 to destination node 10 under the DVP approach.

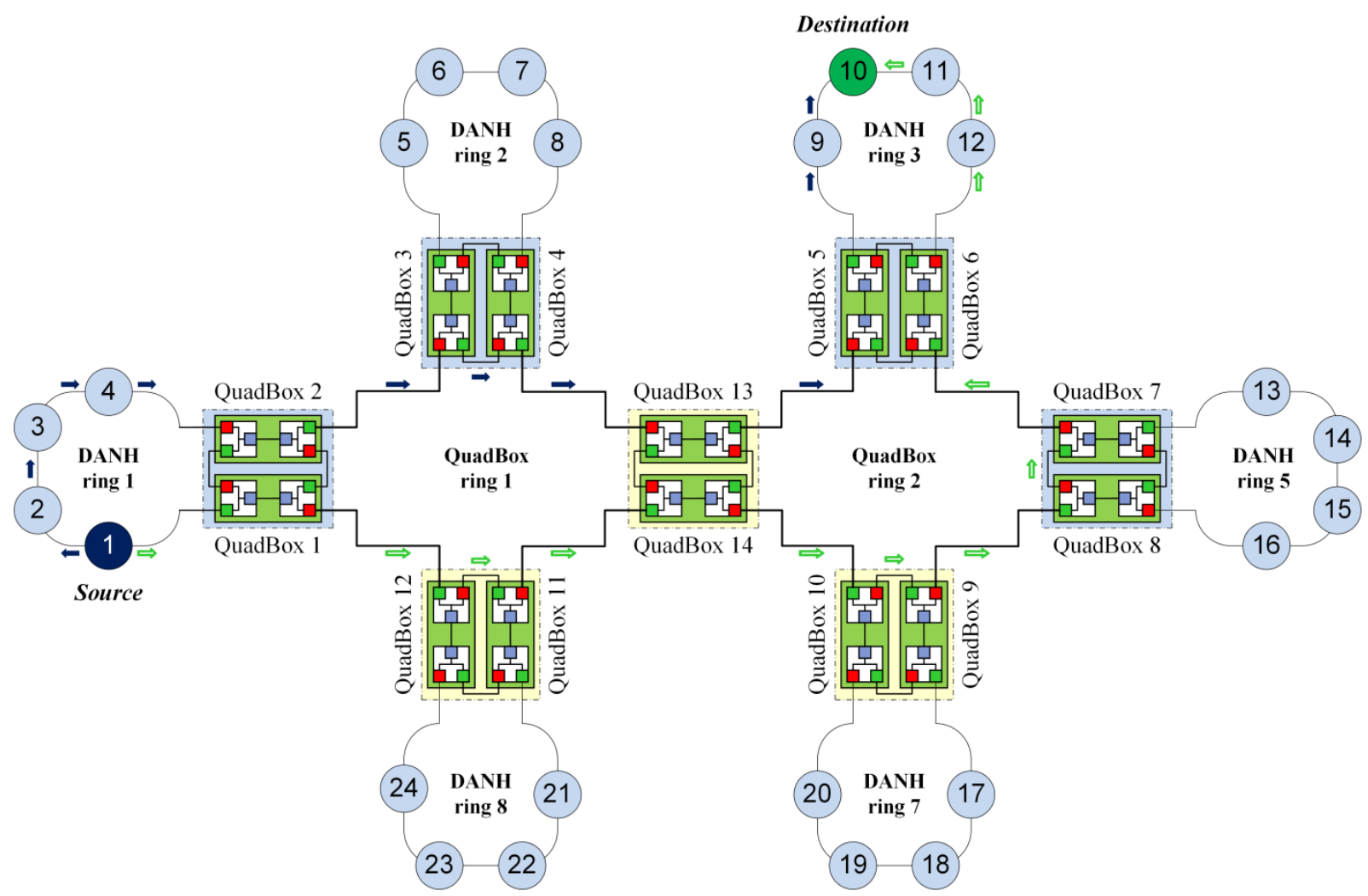

Figure 15. Forwarding unicast traffic under the DVP approach.

For the connection pair of DANH nodes 1 and 10, the DVP approach established two virtual paths, including the first path of DANH 2-DANH 3-DANH 4-QuadBox 2-QuadBox 3-QuadBox 4-QuadBox 13-QuadBox 5-DANH 9, and the second path of QuadBox 1-QuadBox 12-QuadBox 11-QuadBox 14-QuadBox 10-QuadBox 9-QuadBox 8-QuadBox 7- QuadBox 6-DANH 12-DANH 11. Each of these paths transfers only one frame copy from source node 1 to destination node 10, without duplication and flooding, because QuadBox nodes in between these paths forward the frames to 
destination node 10 using their FP table instead of duplicating and flooding the frames as in the standard HSR protocol.

\subsubsection{Advantages and Disadvantages}

Like ODP, DVP significantly reduces redundant unicast traffic compared with the standard HSR protocol by forwarding the unicast traffic through two predefined paths instead of duplicating and flooding in overall network. Instead of building and maintaining the link metric table for building dual paths as in the ODP approach, the DVP approach establishes dual virtual paths for each connection pair of terminal nodes by sending and receiving control messages.

However, since the DVP approach establishes dual paths for each connection pair of terminal nodes, the number of connection pairs is very large. The connection pairs required to build dual paths is equal to $(n \times(n-1)) / 2$ for a network with $n$ terminal nodes. This results in high control overhead for discovering and building dual paths in HSR networks.

\subsection{Ring-Based Dual Paths (RDP)}

\subsubsection{RDP Concepts}

As a predefined path-based technique, RDP [20] establishes two predefined paths for each connection pair and use them for point-to-point communications instead of duplicating and flooding as in the standard HSR protocol. However, unlike ODP and DVP that establish dual paths for each connection pair of terminal nodes, RDP builds dual paths for each connection pair of terminal rings (DANH rings). In the RDP approach, dual paths are ring-based dual paths. The RDP approach forwards unicast frames from a source node to a destination node through the dual paths pre-established between the source DANH ring and the destination DANH ring.

In the RDP approach, the functions of discovering and establishing dual paths are performed only at QuadBoxes, not terminal nodes, such as DANH nodes and RedBox nodes. The RDP approach defines two types of QuadBox nodes: access QuadBoxes and trunk QuadBoxes. Access QuadBoxes connect to at least one DANH ring, while trunk QuadBoxes connect to two QuadBox rings. Like DVP, the RDP approach uses several control messages, such path request (PREQ) and path reply (PREP) message in order to discover and build dual paths for each connection pair of DANH rings.

\subsubsection{RDP Operations}

The functions of discovering and establishing dual paths between DANH rings are implemented at QuadBox nodes. RDP first builds a MAC table for each access QuadBox that connects to a DANH ring, then discovers and establishes dual paths for each connection pair of DANH rings, and, finally, sets up the ring-based dual paths by building a forwarding table for each trunk QuadBox.

(a) Building the MAC table: Each access QuadBox periodically sends a Hello message into its DANH ring. When a DANH node in the ring receives a Hello message, it replies with an Acknowledgment (ACK) message, and then forwards the received Hello message to other DANH nodes in the DANH ring. Each access QuadBox builds its MAC table based on received 
ACK messages from DANH nodes in its DANH ring. When an access QuadBox receives an ACK message, it checks if its MAC table contains the source MAC address of the ACK message. If not, the QuadBox adds the source MAC address into its MAC table. If so, the ACK message is discarded. By learning the MAC addresses of DANH nodes, each access QuadBox learns the MAC addresses of all the DANH nodes connected to its DANH ring. Based on the MAC table, an access QuadBox will not forward a unicast frame into its DANH ring if its MAC table does not contain the destination MAC address of the frame.

(b) Establishing ring-based dual paths: The functions of discovering and establishing dual paths between DANH rings are performed at access QuadBox nodes through a two-way handshake process: path request and path reply.

- Path request: To discover and establish ring-based dual paths for each connection pair of DANH rings, each access QuadBox sends a path request to all of the other access QuadBoxes by broadcasting a path request (PREQ) message. The PREQ message contains information of a sequence number, the source ring ID, the MAC table of the access QuadBox, and a node list that contains ID of QuadBoxes through which the PREQ message has been passed. When a trunk QuadBox receives a PREQ message, it updates its ring table based on the MAC table of the PREQ message. Each entry of the ring table consists of a MAC address of a DANH node and a ring ID of a DANH ring to which the DANH node is being connected. After updating the ring table, the trunk QuadBox adds itself to the node list of the PREQ message and then sends the updated PREQ messages over all of its ports, except the received port. When an access QuadBox node receives a PREQ message, it replies to the path request by sending a path reply (PREP) message, and then adds itself to the node list of the PREQ message and forwards the updated PREQ messages to QuadBox nodes.

- Path reply: When an access QuadBox has received a PREQ message sent by another access QuadBox, it builds a path between the sending and receiving access QuadBox nodes based on the node list of the PREQ message. The path is also a ring-based path between the source ring to which the sending QuadBox is connecting and the destination ring to which the receiving QuadBox is connecting. The receiving QuadBox then responds to the path request by sending a PREP message to the sending QuadBox. The PREP message is generated based on information in the received PREQ message. The PREP message contains information of a sequence number, the source ring ID, the destination ring ID, and a ring-based path between the source and destination rings built based on the node list of the received PREQ message. To reduce path setup overhead for each connection pair of DANH rings, only the access QuadBox that connects to the DANH ring with the lower ring ID sends the PREP message.

(c) Building the forwarding table: Based on received PREQ and PREP messages, trunk QuadBoxes in between ring-based paths build their forwarding table. Each entry of the forwarding table consists of a source ring ID, a destination ring ID, and an output port. When a trunk QuadBox receives a PREQ of an access QuadBox node, it notes the received port associated with the source ring ID of the PREQ message. When the trunk QuadBox receives a PREP message, 
it adds two route entries of the connection pair, one for each direction, to its forwarding table. Since RDP uses two paired QuadBox nodes to connect rings in order to avoid a single point of failure, there are two ring-based paths discovered and established for each connection pair of DANH rings.

Figure 16 shows the process of forwarding unicast frames from source node 1 to destination node 10 under the RDP approach.

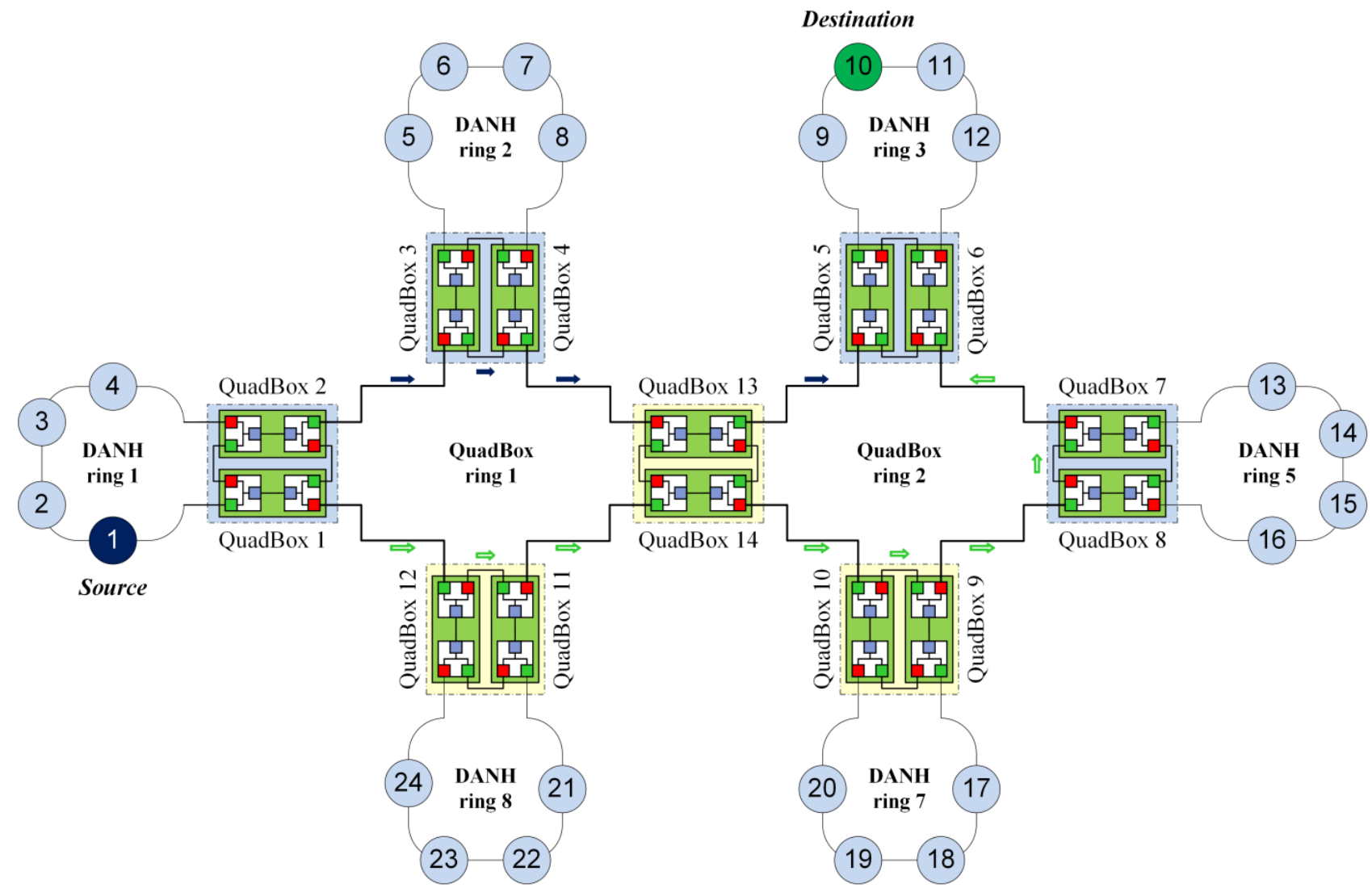

Figure 16. Forwarding unicast traffic under the RDP approach.

In this case, RDP has discovered and established ring-based dual paths without any common nodes for the connection pair of DANH rings 1 and 3. The dual path consists of two ring-based paths, including the first ring-based path of QuadBox 2-QuadBox 3-QuadBox 4-QuadBox 13-QuadBox 5, and the second ring-based path of QuadBox 1-QuadBox 12-QuadBox 11-QuadBox 14-QuadBox 10QuadBox 9-QuadBox 8-QuadBox 7-QuadBox 6. The source node 1 sends two copies of the frame simultaneously through each port in both directions in DANH ring 1. The first copy is forwarded to access QuadBox 2, and the second copy is forwarded to access QuadBox 1. The first and second copies then is forwarded through the ring-based dual paths and arrived to destination DANH ring 3 at access QuadBoxes 5 and 6, respectively. These access QuadBoxes lookup their MAC table and forward the copies to destination DANH ring 3. Finally, the destination node 10 receives two copies of the frame from both directions of DANH ring 3, passes the first frame to its upper layers, and discards the duplicate. 


\subsubsection{Advantages and Disadvantages}

Like other predefined path-based techniques such as ODP and DVP, the RDP approach significantly reduces redundant unicast traffic in HSR networks compared with the standard HSR protocol by forwarding the unicast traffic through two predefined paths. Like DVP, the RDP approach establishes dual paths by sending and receiving control messages, such as PREQ and PREP messages. However, unlike the ODP and DVP approaches that discover and establish node-based dual paths for each connection pair of terminal nodes, the RDP approach discovers and builds dual paths for each connection pair of DANH rings (terminal rings), therefore, the RDP significantly reduces the number of connection pairs, which in turn reduces additional control overhead for discovering and establishing dual paths compared with the ODP and RDP approaches. However, because of using control messages to discover and build dual paths, the RDP approach still has additional control overhead to setup dual paths in HSR networks.

\subsection{Comparison}

We described three predefined path-based techniques for reducing HSR unicast traffic. Characteristics of these techniques are shown in Table 4.

Table 4. Characteristics of predefined path-based techniques.

\begin{tabular}{cccc}
\hline Characteristics & ODP & DVP & RDP \\
\hline Type of connection pair to setup dual paths & Node & Node & Ring \\
Using network topology database & Yes & No & No \\
Using control messages to setup dual paths & No & Yes & Yes \\
Pruning traffic for unused rings & Yes & Yes & Yes \\
Removing traffic circulation & Yes & Yes & Yes \\
Type of filtered traffic & Unicast & Unicast & Unicast \\
Network topology & Any & Any & Ring \\
\hline
\end{tabular}

The ODP approach establishes dual paths based on the network's link information, whereas the DVP and RDP approaches build dual paths by sending and receiving control messages. The ODP and DVP approaches setup node-based dual paths for each connection pair of terminal nodes such as DANH nodes, RedBox nodes, while the RDP establishes ring-based dual paths for each connection pair of DANH rings or terminal rings. Therefore, the RDP approach significantly reduces the number of connection pairs required to discover and establish dual paths.

We consider a sample network consisting of three QuadBox rings and eight DANH rings; each DANH ring has four DANH nodes, as shown in Figure 12. Two paired QuadBoxes are used to connect two rings. The number of DANH nodes in the network is 32 nodes. Under the ODP and DVP approaches, the number of connection pairs of DANH nodes is $32 \times(32-1) / 2=496$ connection pairs. Under the RDP approach, the number of connection pairs of DANH rings is $8 \times(8-1) / 2=28$ connection pairs. Generally, the RDP approach has about $m^{2}$ times less the number of connection pairs than the ODP and DVP approaches, where $m$ is the number of terminal nodes in a ring. Therefore, the RDP significantly reduces the control overhead for discovering and establishing dual paths, as well as the memory space required to store the dual paths compared with the ODP and DVP approaches. 
To compare traffic performance of these techniques, we conducted several simulations using the $\mathrm{OMNeT}++$ simulation tool. We consider the sample network as shown in Figure 12. In these simulations, a source in DANH ring 1 sends unicast frames to a destination in DANH ring 5.

Network unicast frames recorded from the simulations under the standard HSR protocol, the ODP approach, the DVP approach, and the RDP approach are shown in Table 3. Figure 13 shows the comparison of traffic performance of these techniques. The simulation results show that, in the sample network, the predefined path-based techniques have the same network unicast traffic performance. These techniques significantly reduce network unicast traffic by about $80 \%$ compared with standard HSR protocol.

\section{Conclusions}

The standard HSR protocol generates too much unnecessary redundant unicast traffic in HSR networks, resulting in the degradation of network performance. Several techniques have been proposed to solve this problem. In this paper, we presented a review of typical HSR traffic reduction techniques. We showed that these HSR traffic reduction techniques significantly reduce redundant unicast traffic compared to the standard HSR protocol and improve network performance in HSR networks. These techniques are classified into two categories based on their traffic reduction manner, consisting of traffic filtering-based techniques and predefined path-based techniques. Each technique has advantages and disadvantages. The selection of which HSR traffic reduction technique to implement depends on the particular application and trade-offs. Some of the objectives are traffic performance, network topology, control traffic volume, and storage requirements. With this review paper, researchers can acquire what has been investigated, and network designers can identify which technique to use, and what are the trade-offs.

\section{Acknowledgments}

This work was supported by basic science research program through the National Research Foundation of Korea (NRF) funded by the Ministry of Science, Information and Communications Technologies (ICT), and Future Planning (No. 2013R1A1A2008406) and by the Human Resources Development Program of the Korea Institute of Energy Technology Evaluation and Planning (KETEP) grant funded by the Korea government Ministry of Trade, Industry, and Energy (No. 20134030200310).

\section{Author Contributions}

Authors Nguyen Xuan Tien and Jong Myung Rhee conceived and developed the ideas behind the research. Nguyen Xuan Tien carried out the performance analysis and simulations, and wrote the paper under supervision of Jong Myung Rhee. Saad Allawi Nsaif and Jong Myung Rhee reviewed and revised the paper. Jong Myung Rhee supervised the research and finalized the paper.

\section{Conflicts of Interest}

The authors declare no conflict of interest. 


\section{References}

1. Industrial Communication Networks-High-Availability Automation Networks, Part 3: Parallel Redundancy Protocol (PRP) and High-Availability Seamless Redundancy (HSR); IEC 62439-3; International Electrotechnical Commission (IEC): Geneva, Switzerland, 2012.

2. Network Engineering Guidelines for Communication Networks and Systems in Substations; IEC 61850-90-4; International Electrotechnical Commission (IEC): Geneva, Switzerland, 2013.

3. Industrial Communication Networks-High-Availability Automation Networks, Part 1: General Concepts and Calculation Methods; IEC 62439-1; International Electrotechnical Commission (IEC): Geneva, Switzerland, 2010.

4. Kirrmann, H.; Kleineberg, O. HSR: Zero Recovery time and low-cost redundancy for industrial Ethernet (High availability Seamless Redundancy, IEC 62439-3). In Proceedings of the IEEE Conference on Emerging Technologies and Factory Automation (ETFA), Mallorca, Spain, 22-25 September 2009.

5. Kirrmann, H.; Kleineberg, O. Seamless and low-cost redundancy for substation automation systems (High availability Seamless Redundancy, HSR). In Proceedings of the Power and Energy Society General Meeting, Detroit, MI, USA, 24-29 July 2011.

6. Heine, H.; Kleineberg, O. The high-availability seamless redundancy protocol (HSR): Robust fault-tolerant networking and loop prevention through duplicate discard. In Proceedings of the 9th IEEE International Workshop on Factory Communication Systems (WFCS), Lemgo, Germany, 21-24 May 2012.

7. Araujo, J.A.; Lazaro, J.; Astarloa, A.; Zuloaga, A.; Garcia, A. High availability automation networks: PRP and HSR ring implementations. In Proceedings of the IEEE International Symposium on Industrial Electronics (ISIE), Hangzhou, China, 28-31 May 2012.

8. Nsaif, S.A.; Rhee, J.M. Improvement of high-availability seamless redundancy (HSR) traffic performance. In Proceedings of the 14th International Conference on Advanced Communication Technology (ICACT), Gangwon, Korea, 7-10 February 2012.

9. Nsaif, S.A.; Rhee, J.M. Improvement of High-Availability Seamless Redundancy HSR Traffic Performance for Smart Grid Communications. J. Commun. Netw. 2012, 14, 653-661.

10. Rhee, J.M.; Nsaif, S.A. Quick Removing (QR) Approach. In Traffic Reduction Algorithms for High-Availability Seamless Redundancy (HSR) Protocol; Myongji Research Institute: Yongin, Korea, 2014; pp. 21-32.

11. Rhee, J.M.; Nsaif, S.A. High-Availability Seamless Redundancy (HSR) Protocol for Smart Grid communications. In Smart Grids Technologies, Applications and Management Systems; Nova Science Publishers: New York, NY, USA, 2014; pp. 185-234.

12. Shin, M.; Joe, I. Performance improvement for the HSR ring protocol with traffic control in smart grid. In Proceedings of the Future Generation Information Technology (FGIT) 2012, Gangwon, Korea, 16-19 December 2012.

13. Abdulsalam, I.R.; Rhee, J.M. Improvement of high-availability seamless redundancy (HSR) unicast traffic performance using port locking. In Proceedings of the Fourth World Congress on Software Engineering, Hong Kong, China, 3-4 December 2013. 
14. Rhee, J.M.; Nsaif, S.A. Port Locking (PL) Approach. In Traffic Reduction Algorithms for High-Availability Seamless Redundancy (HSR) Protocol; Myongji Research Institute: Yongin, Korea, 2014; pp. 131-140.

15. Altaha, I.R.; Rhee, J.M.; Pham H.A. Improvement of high-availability seamless redundancy (HSR) unicast traffic performance using enhanced port locking (EPL) approach. IEICE Trans. Inform. Syst. 2015, 98, 1646-1656.

16. Tien, N.X.; Rhee, J.M. FHT: A Novel approach for filtering high-availability seamless redundancy (HSR) traffic. Energies 2015, 8, 6249-6274.

17. Tien, N.X.; Nsaif, S.A.; Rhee, J.M. High-availability seamless redundancy (HSR) traffic reduction using optimal dual paths (ODP). In Proceedings of the International Conference on Green and Human Information Technology (ICGHIT), Da Nang, Vietnam, 4-6 February 2015.

18. Nsaif, S.A.; Rhee, J.M. DVP: A novel high-availability seamless redundancy (HSR) protocol traffic-reduction algorithm for a substation automation system network. Energies 2014, 7, 1792-1810.

19. Rhee, J.M.; Nsaif, S.A. Dual Virtual Paths (DVP) Algorithm. In Traffic Reduction Algorithms for High-Availability Seamless Redundancy (HSR) Protocol; Myongji Research Institute: Yongin, Korea, 2014; pp. 49-110.

20. Tien, N.X.; Rhee, J.M. Reducing high-availability seamless redundancy (HSR) unicast traffic using ring-based dual paths (RDP). J. Commun. Netw. 2015, under review.

21. IEEE 802.3-IEEE Standards for Ethernet. Available online: http://www.ieee802.org/3/ (accessed on 30 June 2015).

22. Song, S.; Huang, J.; Kappler, P.; Freimark, R.; Kozlik, T. Fault-tolerant Ethernet middleware for IP-based process control networks. In Proceedings of the 25th Annual IEEE Conference on Local Computer Networks, Tampa, FL, USA, 8-12 November 2000.

23. Lee, D.H.; Cho, Y.Z.; Pham, H.A.; Rhee, J.M.; Ryu, Y.S. SAFE: A scalable autonomous fault tolerant Ethernet scheme for large-scale star network. IEICE Trans. Commun. 2012, 95, 3158-3167.

24. Pham, H.A.; Rhee, J.M. RSAFE: A robust software-based fault-tolerant scheme for large-scale Ethernet networks. J. Inf. Sci. Eng. 2015, 31, 399-413.

25. IEEE 802.1D-1998-IEEE Standard for Local Area Networks: Media Access Control (MAC) Bridges. Available online: http://www.ieee802.org/1/pages/802.1D.html (accessed on 30 June 2015).

26. IEEE 802.1D-2004-IEEE Standard for Local and Metropolitan Area Networks: Media Access Control (MAC) Bridges. Available online: http://www.ieee802.org/1/pages/802.1D-2003.html (accessed on 30 June 2015).

27. IEEE 802.1aq Standard-Shortest Path Bridging. Available online: http://www.ieee802. org/1/pages/802.1aq.html (accessed on 10 September 2015).

28. IETF RFC 6325-Routing Bridges (RBridges): Base Protocol Specification. Available online: https://tools.ietf.org/html/rfc6325 (accessed on 10 September 2015).

29. IETF RFC 6326-Transparent Interconnection of Lots of Links (TRILL) Use of IS-IS. Available online: https://tools.ietf.org/html/rfc6326 (accessed on 10 September 2015).

30. IETF RFC 6327-Routing Bridges (RBridges): Adjacency. Available online: https://tools.ietf.org/ html/rfc6327 (accessed on 10 September 2015). 
31. IETF RFC 6361-PPP Transparent Interconnection of Lots of Links (TRILL) Protocol Control Protocol. Available online: https://tools.ietf.org/html/rfc6361 (accessed on 10 September 2015).

32. IETF RFC 6439-Routing Bridges (RBridges): Appointed Forwarders. Available online: https://tools.ietf.org/html/rfc6439 (accessed on 10 September 2015).

33. IEEE 802.1ak Standard-Multiple Registration Protocol. Available online: http://www.ieee802. org/1/pages/802.1ak.html (accessed on 10 September 2015).

34. Industrial Communication Networks: High-Availability Automation Networks, Part 2: Media Redundancy Protocol (MRP); IEC 62439-2; International Electrotechnical Commission (IEC): Geneva, Switzerland, 2010.

35. Industrial Communication Networks: High-Availability Automation Networks, Part 4: Cross-Network Redundancy Protocol (CRP); IEC 62439-4; International Electrotechnical Commission (IEC): Geneva, Switzerland, 2010.

36. Industrial Communication Networks: High-Availability Automation Networks, Part 5: Beacon Redundancy Protocol (BRP); IEC 62439-5; International Electrotechnical Commission (IEC): Geneva, Switzerland, 2010.

37. OMNeT++ Version 4.6 Simulator. Available online: http://www.omnetpp.org/ (accessed on 15 January 2015).

(C) 2015 by the authors; licensee MDPI, Basel, Switzerland. This article is an open access article distributed under the terms and conditions of the Creative Commons Attribution license (http://creativecommons.org/licenses/by/4.0/). 